\title{
Equações de Difusão Não Locais do tipo Neumann
}

\author{
Allan Fernandes Banzatto \\ DiSSERTAÇÃO APRESENTADA \\ $\mathrm{AO}$ \\ Instituto DE MATEMÁtica E EstatísticA \\ DA \\ UNIVERSIDADE DE SÃO PAUlO \\ PARA \\ OBTENÇÃO DO TÍTULO \\ DE \\ Mestre EM CiênCIAS
}

Programa: Programa de Pós Graduação em Matemática Aplicada Orientador: Prof. Dr. Marcone Corrêa Pereira

Durante o desenvolvimento deste trabalho o autor recebeu auxílio financeiro da CNPq

São Paulo, agosto de 2018 


\section{Equações de Difusão Não Locais do tipo Neumann}

Esta versão da dissertação contém as correções e alterações sugeridas pela Comissão Julgadora durante a defesa da versão original do trabalho, realizada em 27/09/2018. Uma cópia da versão original está disponível no

Instituto de Matemática e Estatística da Universidade de São Paulo.

Comissão Julgadora:

- Prof. Dr. Marcone Corrêa Pereira (orientador) - IME-USP

- Prof ${ }^{a}$. Dr ${ }^{a}$. Juliana Fernandes da Silva Pimentel - UFRJ

- Prof. Dr. Marcus Antonio Mendonça Marrocos - UFABC 


\section{Agradecimentos}

Agradeço ao meu orientador, Professor Marcone Corrêa Pereira, pela paciência e orientação, à minha família pelo apoio e suporte, a todos os meus amigos da Pós-Graduação do IME-USP pela força e ajuda e também agradeço à Letícia, pois sem ela este trabalho não teria chegado ao fim. 


\section{Resumo}

Banzatto, A. F. Equações de Difusão Não Locais do tipo Neumann. 2018. Dissertação (Mestrado) - Instituto de Matemática e Estatística, Universidade de São Paulo, São Paulo, 2018.

Neste trabalho estudaremos uma classe de problemas não locais do tipo Neumann. Consideramos o caso linear não homogêneo, bem como o semi-linear com não linearidades globalmente Lipschitz. Procuramos escrever um trabalho auto-contido. Apresentamos alguns resultados clássicos de Análise e suas aplicações no contexto de equação de evolução não local. Na introdução, apresentamos uma motivação para tais equações tendo em vista os fenômenos de reação e difusão baseados no trabalho de P. Fife [4].

Palavras-chave: EDP, Difusão, Não homogênea, Neumann. 


\section{Abstract}

Banzatto, A. F. Neumann Non-Local Diffusion Equations. 2018. Dissertação (Mestrado) Instituto de Matemática e Estatística, Universidade de São Paulo, São Paulo, 2018.

In this work we will study a class of nonlocal problems of the Neumann type. We consider the non-homogeneous linear case as well as the semi-linear one with globally Lipschitz non-linearities. We seek to write a self-contained work with some classic results of Analysis and its applications in the context of non-local evolution equations. In the introduction, we present a motivation for such equations in view of the phenomena of reaction and diffusion based on the work of P. Fife [4].

Keywords: PDE, Diffusion, Nonhomogeneous, Neumann. 


\section{Sumário}

1 Resultados Preliminares $\quad 5$

1.1 Definições Básicas e Desigualdades . . . . . . . . . . . . . . . . . . 5

1.2 Teorema de Arzelá-Ascoli . . . . . . . . . . . . . . . . . . . . 7

1.3 O Espectro de um Operador Limitado . . . . . . . . . . . . . . . . . . . . 9

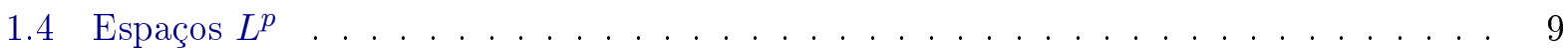

2 Contração Com Parâmetros e o Teorema de Stampacchia $\quad 13$

2.1 Teorema da Contração Uniforme . . . . . . . . . . . . . . . . . . . . . 13

2.2 Teorema de Stampacchia e Lax-Milgram . . . . . . . . . . . . . . . . . 18

3 Problema de Neumann Não Local $\quad 29$

3.1 Equação Linear Não Homogênea . . . . . . . . . . . . . . . . . . . . . . . . . . . 29

3.1.1 Resultados Preliminares . . . . . . . . . . . . . . . . . . 29

3.1 .2 Existência e Unicidade . . . . . . . . . . . . . . . . . . . . . . . . . 38

3.2 Uma Equação Semi-Linear . . . . . . . . . . . . . . . . . . . . . . . . 44

3.3 Regularidade com Relação aos Parâmetros . . . . . . . . . . . . . . . . . . . . . 47

$\begin{array}{ll}\text { Referências Bibliográficas } & 51\end{array}$ 
viii SUMÁRIO 


\section{Introdução}

Baseando-se principalmente nos trabalhos [1, 10], a finalidade desta dissertação é discutir alguns resultados sobre equações de evolução semi-lineares não locais do tipo Neumann. Lidaremos com a unicidade e existência global de soluções e comportamento assintótico no caso linear. Inicialmente, utilizaremos algumas ideias de [4] para dar uma motivação da classe de equações discutidas em nosso trabalho.

Para isto, considere $J: \mathbb{R}^{n} \longrightarrow \mathbb{R}$ uma função par, não negativa e contínua com

$$
\int_{\mathbb{R}^{n}} J(x) d x=1 \text { e }\|J\|_{L^{\infty}\left(\mathbb{R}^{n}\right)}<\infty .
$$

Inicialmente, observamos que equações de evolução não locais da forma

$$
\left\{\begin{array}{l}
u_{t}(t, x)=(J * u-u)(t, x)=\int_{\mathbb{R}^{n}} J(x-y) u(t, y) d y-u(t, x), x \in \Omega, t>0 \\
u(t, x) \equiv 0, \text { em } \mathbb{R}^{N} \backslash \Omega \\
u(0, x)=u_{0}(x),
\end{array}\right.
$$

bem como suas variações, tem sido muito utilizadas na modelagem de processos de difusão. De acordo com [4], se $u(t, x)$ representa a densidade populacional no ponto $x$ e instante $t$, e $J(x-y)$ a distribuição probabilística de nos movermos do ponto $y$ para o ponto $x$, então

$$
\int_{\mathbb{R}^{N}} J(x-y) u(t, y) d y=(J * u)(t, x)
$$

é a taxa cujo indivíduos estão chegando na posição $x$ de todos os outros lugares e

$$
u(t, x)=\int_{\mathbb{R}^{N}} J(y-x) u(t, x) d y
$$

é a taxa que mostra o deslocamento de $x$ para todos os outros lugares $y$. Esta consideração, na falta de fontes internas ou externas e na suposição de que fora de $\Omega$ não há vida, nos leva para o fato de que a densidade $u$ satisfaz a equação (1).

O problema (1) é chamado de equação de difusão não local do tipo Dirichlet, embora a difusão da densidade $u$ no ponto $x$ e tempo $t$ não dependa das derivadas $u(t, x)$, ela depende de todos os valores de $u$ numa vizinhança de $x$ através do termo de convolução $J * u$ bem como dos valores de $u$ em $x$.

Fixemos então $\Omega$ um domínio limitado e conexo do $\mathbb{R}^{N}$. Vamos começar a olhar para possíveis generalizações da equação clássica semilinear do calor

$$
u_{t}(t, x)=\Delta u(t, x)-f(u(t, x)), \forall x \in \Omega \text { e } t>0 .
$$

Focando em extensões não locais, devemos questionar qual é a contrapartida mais "natural"do termo de difusão $\Delta u$. Uma maneira de motivar nossa resposta é caracterizar seu negativo, $-\Delta u$ como a derivada funcional no espaço $L^{2}(\Omega)$ da integral de Dirichlet

$$
\epsilon_{0}[u(t, x)]=\int_{\Omega} \frac{1}{2}|\nabla u(t, x)|^{2} d x .
$$


Lembremos da seguinte relação muito utilizada em cálculo:

$$
\operatorname{div}\left(u_{t}(t, x) \nabla u(t, x)\right)=\nabla u_{t}(t, x) \nabla u(t, x)+u_{t}(t, x) \Delta u(t, x),
$$

ou seja,

$$
\nabla u_{t}(t, x) \nabla u(t, x)=\operatorname{div}\left(u_{t}(t, x) \nabla u(t, x)\right)-u_{t}(t, x) \Delta u(t, x) .
$$

Então, para $u$ suficientemente suave, obtemos

$$
\frac{d \epsilon_{0}}{d t}[u(t, \cdot)]=\int_{\Omega} \nabla u_{t}(t, \cdot) \nabla u(t, \cdot) d x .
$$

Utilizando (5) e assumindo que $\int_{\Omega} \operatorname{div}(u(t, x) \nabla u(t, x)) d x=0$ neste caso, encontramos

$$
\frac{d \epsilon_{0}}{d t}[u(t, \cdot)]=\int_{\Omega}-u_{t}(t, \cdot) \Delta u(t, \cdot) d x
$$

ou seja,

$$
\frac{d \epsilon_{0}}{d t}[u(t, \cdot)]=\left\langle-\Delta u(t, \cdot), u_{t}(t, \cdot)\right\rangle,
$$

onde a operação $\langle\cdot, \cdot\rangle$ é o produto interno de $L^{2}(\Omega)$. Assim, escrevemos formalmente que

$$
-\Delta u(t, x)=\frac{\delta \epsilon_{0}}{\delta u}
$$

onde $\frac{\delta \epsilon}{\delta u}$ é o diferencial em $L^{2}(\Omega)$ do funcional $\epsilon[u(t, x)]$.

A energia $\epsilon_{0}$ é muitas vezes interpretada como uma medida de quanto $u$ deixa de ser constante. Além disso, para todo $u \in H^{1}(\Omega), \epsilon_{0}[u] \geq 0$ com $\epsilon_{0}[u]=0$ se, e somente se, $u$ é constante em quase todo ponto.

Um funcional energético não local natural que pode agir como uma medida alternativa do mesmo problema é

$$
\epsilon_{10}[u(t, x)]=\int_{\mathbb{R}^{n}} \int_{\mathbb{R}^{n}} \frac{1}{4} J(x-y)(u(t, x)-u(t, y))^{2} d x d y,
$$

para alguma função não negativa $J$ assumindo, por exemplo que $u(x) \equiv 0 \mathrm{em} \mathbb{R}^{N} \backslash \Omega$, como em (1). Novamente, $\epsilon_{10}[u] \geq 0$ e $\epsilon_{10}[u]=0$ apenas quando $u$ é constante.

Assim, utilizando a Proposição 3.1, do Capítulo 3, temos que

$$
\begin{aligned}
\frac{d \epsilon_{10}}{d t}[u(t, x)] & =\frac{1}{2} \int_{\mathbb{R}^{N}} \int_{\mathbb{R}^{N}} J(x-y)(u(t, x)-u(t, y))\left(u_{t}(t, x)-u_{t}(t, y)\right) d x d y \\
& =-\int_{\mathbb{R}^{N}} u_{t}(t, y) \int_{\mathbb{R}^{N}} J(x-y)(u(t, x)-u(t, y) d x d y
\end{aligned}
$$

o que nos dá

$$
\frac{\delta \epsilon_{10}}{\delta u}[u(t, x)]=-\int_{\mathbb{R}^{n}} J(x-y)(u(t, x)-u(t, y)) d x,
$$

que é equivalente a

$$
\frac{\delta \epsilon_{10}}{\delta u}[u(t, x)]=-J * u(t, x)+\left(\int_{\mathbb{R}^{n}} J(x-y) d x\right) u(t, x) .
$$

Utilizando a hipótese $\int_{\mathbb{R}^{N}} J(x-y) d x=1$, encontramos

$$
\frac{\delta}{\delta u} \epsilon_{10}(u(t, x))=-J * u(t, x)+u(t, x) .
$$

Dessa forma, se entendemos que $\epsilon_{10}$ é formalmente análogo a $\epsilon_{0}$, concluímos que a expressão $J * u-u$ 
também é análoga a $\Delta u$ em (2).

Indo um pouco além, podemos incorporar o termo não linear $f(u)$ em tais modelos da seguinte maneira: seja $W$ uma função derivável tal que $W^{\prime}=f$. Definimos

$$
\epsilon[u(t, x)]=\epsilon_{0}[u(t, x)]+\int_{\Omega} W(u(t, x)) d x
$$

e

$$
\epsilon_{1}[u(t, x)]=\epsilon_{10}[u(t, x)]+\int_{\Omega} W(u(t, x)) d x .
$$

Observamos que o efeito não linearidade pode ser interpretada como uma reação ou força externa agindo no sistema assumindo valores que dependem da própria densidade $u$ no sistema. (Para maiores discussões neste sentido, veja [8].)

Assim da equação (2) concluímos que

$$
u_{t}(t, x)=-\frac{\delta \epsilon}{\delta u}
$$

já que, por $(7)$ e $W^{\prime}(u)=f(u)$ temos

$$
\frac{\delta}{\delta u} \epsilon[u(t, x)]=\frac{\delta}{\delta u} \epsilon_{0}[u(t, x)]+\frac{\delta}{\delta u} \int_{\Omega} W(u(t, x)) d x=-\Delta u+f(u(t, x)) .
$$

Analogamente, podemos calcular sua versão não local correspondente $u_{t}(t, x)=-\frac{\delta \epsilon_{1}}{\delta u}$ que é

$$
u_{t}(t, x)=J * u(t, x)-u(t, x)-f(u(t, x)) .
$$

A expressão $\frac{\delta \epsilon}{\delta u}$ é o diferencial em $L^{2}(\Omega)$ do funcional $\epsilon[u(t, x)]$, e as leis de evolução (2), (13) e em geral (12) são derivadas de suas respectivas energias. Deste modo, obtemos de (12) que

$$
\frac{d}{d t} \epsilon[u(t, x)]=\left\langle\frac{\delta \epsilon}{\delta u}, u_{t}\right\rangle=-\left\|\frac{\delta \epsilon}{\delta u}\right\|^{2} .
$$

Outra interpretação de (12) e seu análogo para $\epsilon_{1}$ sugere o seguinte: do lado direito, a quantidade $-\frac{\delta \epsilon}{\delta u}$, sendo o negativo da taxa a qual $\epsilon$ muda quando submetida a variação de $u$, pode ser considerada uma força, em analogia com os campos de força potenciais. O lado esquerdo de (12) é então a reação ou fluxo, no sentido de um movimento ou taxa de mudança se contrapondo a uma força exercida. Assim, (12) pode representar alguma relação que envolve força/reação.

Finalmente, nos modelos de difusão de organismos no espaço onde $u$ é sua densidade, a expressão $J * u-u$ pode representar transporte através de mecanismos de reação a longa distância. Com efeito, se $J(r)$ é uma função de distribuição probabilística de difusão em relação à distância $r$, temos que a expressão $J * u-u$ à direita de (13) representa a taxa líquida de aumento devido à difusão. Note que existe um princípio de conservação, já que

$$
\int_{\mathbb{R}^{N}}(J * u(t, x)-u(t, x)) d x=0 .
$$

Para problemas locais, as duas condições de contorno mais comuns são a de Neumann e Dirichlet. Quando olhamos para condições de contorno em problemas não locais, temos que modificar as formulações usuais das equações locais. O problema que daremos atenção neste trabalho é o análogo às condições de Neumann:

$$
\left\{\begin{array}{l}
u_{t}(t, x)=\int_{\Omega} J(x-y)(u(t, y)-u(t, x)) d y, x \in \Omega, t>0 \\
u(0, x)=u_{0}(x), x \in \Omega .
\end{array}\right.
$$

Neste modelo temos que o integrando leva em conta a difusão apenas dentro de $\Omega$. De fato, como 
já discutido anteriormente, temos que a expressão $\int J(x-y)(u(t, y)-u(t, x)) d y$ leva em conta os indivíduos chegando ou saindo da posição $x$ de outros lugares. Como estamos integrando em $\Omega$, impomos que a difusão se estabeleça apenas em $\Omega$. Desta forma, os indivíduos não podem entrar ou sair da região de interesse.

Problemas não locais como (1) e (15) são frequentemente utilizados para modelar processos ou situações em ecologia, biologia, processamento de imagens, sistemas de partículas, etc. ${ }^{1}$

Finalmente observamos que aqui nesta dissertação, também vamos considerar o problema semilinear relacionado a (15)

$$
\left\{\begin{array}{l}
u_{t}(t, x)=\int_{\Omega} J(x-y)(u(t, y)-u(t, x)) d y+f(u(t, x)), \\
u(0, x)=u_{0}(x)
\end{array}\right.
$$

assumindo $f$ globalmente Lipschitz em $\mathbb{R}$. Assim como no problema (15), temos que a expressão $\int J(x-y)(u(t, y)-u(t, x) d y$ leva em conta os indivíduos chegando ou saindo da posição $x$ de outros lugares, com a difusão passando-se apenas em $\Omega$, sobre ação de uma força externa que depende da própria solução $u$. Ou seja, podemos caracterizar $f$ como sendo um agente externo que altera de alguma forma o modo como os indivíduos se locomovem e interagem dentro de $\Omega$. Para tal problema também mostraremos unicidade e existência global de soluções em $L^{2}(\Omega)$.

\footnotetext{
${ }^{1}$ Veja por exemplo [1, 4]. Nesse sentido o estudo qualitativo destes modelos se torna uma tarefa útil e importante em Análise Matemática.
} 


\section{Capítulo 1}

\section{Resultados Preliminares}

Neste capítulo vamos enunciar e demonstrar alguns resultados que serão usados no decorrer deste trabalho. Como por exemplo mencionamos as Desigualdades de Young, Hölder e Minkowski. Enunciaremos também, baseando-se em [11], o Teorema de Arzelá-Ascoli, que nos será muito útil. Logo após estudaremos Operadores Compactos. Em seguida introduzimos os Espaços $L^{p}$ e concluímos o capítulo com o Teorema de Fischer-Riez, que nos diz que todo espaço $L^{p}$ é um espaço de Banach.

\subsection{Definições Básicas e Desigualdades}

Definição 1.1 Uma sequência $\left\{x_{n}\right\}$ é dita de Cauchy se: dado $\varepsilon>0$, existe $n_{0} \in \mathbb{N}$ tal que, se $n, m>n_{0}$, então

$$
d\left(x_{n}, x_{m}\right)<\varepsilon .
$$

Definição 1.2 Um espaço normado $X$ é um espaço de Banach se toda sequência de Cauchy em $X$ converge. Dizemos que $\mathcal{L}(X, Y)$ é o espaço de operadores lineares continuos com dominio $X e$ contra-dominio $Y$.

Definição 1.3 Um espaço de Hilbert é um espaço vetorial com produto interno que também é um espaço de Banach com a norma canônica definida pelo produto interno

$$
\|x\|=\sqrt{\langle x, x\rangle}
$$

Notação: Escrevemos $f(x)=O(x)$ se $x \rightarrow x_{0}$, caso exista $c>0$ tal que $|f(x)| \leq c|g(x)|$, para todo $x$ próximo a $x_{0}$ e, $f(x)=o(g(x))$, se $x \rightarrow x_{0}$, caso ocorra

$$
\lim _{x \rightarrow x_{0}} \frac{f(x)}{g(x)}=0
$$

Definição 1.4 Uma aplicação $f: X \longrightarrow Y$ é diferenciável no ponto a $\in X$ se existe uma aplicação continua linear $f^{\prime}(a): X \longrightarrow Y$ tal que

$$
\left\|f(x)-f(a)-f^{\prime}(a)(x-a)\right\|_{Y}=o\left(\|x-a\|_{X}\right), \operatorname{com} x \rightarrow a,
$$

ou seja,

$$
\lim _{x \rightarrow a} \frac{\left\|f(x)-f(a)-f^{\prime}(a)(x-a)\right\|_{Y}}{\|x-a\|_{X}}=0 .
$$

Neste caso, $f^{\prime}(a) \in \mathcal{L}(X, Y)$ é chamada derivada de Frechèt em a.

Quando $f$ é n-vezes continuamente diferenciável em um aberto $U \subset X$, consideremos suas derivadas de k-ésima ordem $f^{(k)}, 1 \leq k \leq n$ como sendo um polinômio contínuo de ordem $k$ para cada $x \in U$, determinado pela fórmula de Taylor: 


$$
f(x+h)=\sum_{k=0}^{n} \frac{1}{k !} f^{(k)}(x)\left(h^{k}\right)+o\left(\|h\|_{X}^{n}\right), h \rightarrow 0, x \in U,
$$

onde $f^{(0)}(x)=f(x)$.

Uma aplicação $f: U \subset X \longrightarrow Y, U$ aberto, é analítica em $U$ se $f$ é infinitamente sempre diferenciável em cada ponto de $U$ e, se $\forall x \in U$, existe $\delta=\delta(x)>0$, tal que sempre que $\|h\|_{X} \leq \delta$,

$$
f(x+h)=\sum_{k=0}^{\infty} \frac{1}{k !} f^{(k)}(x)\left(h^{k}\right),
$$

a série convergente na norma $Y$ uniformemente em $\|h\|_{X} \leq \delta$.

Proposição 1.1 Aplicações contínuas com domínios compactos são uniformemente contínuas.

Demonstração: Seja $K \subset \mathbb{R}^{N}$ um conjunto compacto, $Y$ um espaço de Banach, $f: K \longrightarrow Y$ uma aplicação contínua. Sejam $\left\{x_{k}\right\}_{k \in \mathbb{N}},\left\{y_{k}\right\}_{k \in \mathbb{N}} \subset K$ tais que $x_{k}-y_{k} \rightarrow 0$. Suponha que existe $\delta>0$ e subsequência $\left\{k_{1}, \cdots, k_{j}, \cdots\right\}$ tais que

$$
\left\|f\left(x_{k_{j}}\right)-f\left(y_{k_{j}}\right)\right\| \geq \delta, \forall j \in \mathbb{N} .
$$

Como $K$ é compacto, tomando subsequência, se necessário, podemos supor que $\lim _{j \rightarrow \infty} x_{k_{j}}=$ $\lim _{j \rightarrow \infty} y_{k_{j}}=z_{0} \in K$. Mas $f$ é contínua, então $\left\|f\left(x_{k_{j}}\right)-f\left(y_{k_{j}}\right)\right\| \rightarrow 0$. Mostrando que não pode existir tal $\delta>0$ e, assim, $\left\|f\left(x_{k}\right)-f\left(y_{k}\right)\right\| \rightarrow 0$. Portanto $f$ é uniformemente contínua.

Lema 1.2 Para todo $a, b \geq 0$, vale

$$
(a+b)^{p} \leq 2^{p}\left(a^{p}+b^{p}\right) .
$$

Demonstração: De fato, temos

$$
\begin{aligned}
(a+b)^{p} & \leq(\max \{a, b\}+\max \{a, b\})^{p} \\
& =(2 \max \{a, b\})^{p} \\
& =2^{p}\{\max a, b\}^{p} \leq 2^{p}\left(a^{p}+b^{p}\right) .
\end{aligned}
$$

Proposição 1.3 (Desigualdade de Young) Se $a, b \geq 0, p>1 e \frac{1}{p}+\frac{1}{q}=1$ então

$$
a b \leq \frac{a^{p}}{p}+\frac{b^{q}}{q} .
$$

Demonstração: Se $a=0$ e $b=0$, a conclusão é clara. Assumamos que ambos sejam postivos $(a, b>0)$. Se $k \in(0,1)$, definimos $f(t)$ para $t \geq 1$ por $f(t)=k(t-1)-t^{k}+1$. Notemos que $f(1)=k(1-1)-1^{k}+1=0$ e que

$$
\begin{aligned}
f^{\prime}(t) & =k-k t^{k-1} \geq 0 \\
& \Leftrightarrow k\left(1-t^{k-1}\right) \geq 0 \\
& \Leftrightarrow 1-t^{k-1} \geq 0 \\
& \Leftrightarrow t^{k-1} \leq 1 \\
& \Leftrightarrow \frac{1}{t^{1-k}} \leq 1,
\end{aligned}
$$

que vale pois $1-k>0$ e $t \geq 1$. Deste fato $\left(f^{\prime}(t) \geq 0\right)$ concluímos que $f(t)=k(t-1)-t^{k}+1 \geq 0$, para todo $t \geq 1$. Daí,

$$
t^{k} \leq k(t-1)+1=k t+(1-k) .
$$


Se $a \geq b$, colocamos $t=\frac{a}{b}$ (portanto, $t \geq 1$ ) e $k=\frac{1}{p}$ (pertence a $(0,1)$ pois $p \geq 1$ ). Substituindo em (1.1), temos

$$
\left(\frac{a}{b}\right)^{\frac{1}{p}} \leq \frac{1}{p}\left(\frac{a}{b}-1\right)+1
$$

Multiplicando a desigualdade acima por $b^{\frac{1}{q}} b^{\frac{1}{p}}$ temos,

$$
a^{\frac{1}{p}} b^{\frac{1}{q}} \leq \frac{1}{p}\left(\frac{a}{b}-1\right) b^{\frac{1}{q}} b^{\frac{1}{p}}+b^{\frac{1}{q}} b^{\frac{1}{p}}
$$

Usando $\frac{1}{p}+\frac{1}{q}=1$, ainda obtemos

$$
\begin{aligned}
a^{\frac{1}{p}} b^{\frac{1}{q}} & \leq \frac{b}{p}\left(\frac{a}{b}-1\right)+b \\
& =\frac{a}{p}-\frac{b}{p}+b \\
& =\frac{a}{p}+b\left(1-\frac{1}{p}\right) \\
& =\frac{a}{p}+b\left(\frac{1}{q}\right) \\
& =\frac{a}{p}+\frac{b}{q} .
\end{aligned}
$$

O caso $a<b$ é completamente análogo.

\subsection{Teorema de Arzelá-Ascoli}

Definição 1.5 Seja $X$ um espaço topológico. Dizemos que $H \subset X$ é relatimente compacto se $\bar{H}$ é compacto.

Definição 1.6 Seja $X$ um subconjunto de um espaço métrico. Dizemos que $X$ é totalmente limitado se dado $\varepsilon>0$ existe um recobrimento $\left\{O_{1}, \cdots, O_{k}\right\}$ com a propriedade: se $x, y \in O_{i}$, então $d(x, y) \leq$ $\varepsilon$.

Teorema 1.4 (Arzelá-Ascoli) Suponha $X$ um espaço métrico compacto, $C(X, \mathbb{C})$ o conjunto de todas as funçôes $f: X \longrightarrow \mathbb{C}$ contínuas, com a métrica

$$
d(f, g)=\sup \{d(f(x), g(x)) ; x \in X\}
$$

$e \Phi \subset C(X, \mathbb{C})$ é pontualmente limitado e equicontínua. Mais explicitamente

- a) $\sup \{|f(x)|: f \in \Phi\}<\infty \forall x \in X, e$

- b) Se $\varepsilon>0$, todo $x \in X$ tem uma vizinhança $V$ tal que

$$
|f(y)-f(x)|<\varepsilon
$$

para todo $y \in V$ e para toda $f \in \Phi$.

Então $\Phi$ é totalmente limitada em $C(X, \mathbb{C})$.

Demonstração: Fixe $\varepsilon>0$. Para todo $x \in X$, seja $V_{x}$ uma vizinhança tal que b) ocorra, $\cup_{x \in X} V_{x} \supset$ $X$. Como $X$ é compacto, existem pontos $x_{1}, \cdots, x_{n} \in X$, com vizinhanças $V_{1}, \cdots, V_{n}$ tais que $X=\cup_{i=1}^{n} V_{i}$, de modo que

$$
\left|f(x)-f\left(x_{i}\right)\right|<\varepsilon,\left(\forall f \in \Phi, x \in V_{i}, 1 \leq i \leq n\right) .
$$


Note que

$$
|f(x)|=\left|f(x)+f\left(x_{i}\right)-f\left(x_{i}\right)\right| \leq\left|f(x)-f\left(x_{i}\right)\right|+\left|f\left(x_{i}\right)\right|,
$$

e, por a)

$$
|f(x)|<\varepsilon+M_{i}
$$

onde $M_{i}=\sup \left\{\left|f\left(x_{i}\right)\right|: f \in \Phi\right\}$.

Como $X$ é compacto, segue que

$$
\begin{aligned}
\sup \{|f(x)|: x \in X, f \in \Phi\} & =\sup _{i=1}^{n}\left\{|f(x)|: x \in V_{i}, f \in \Phi\right\} \\
& \leq \sum_{i=1}^{n} \sup \left\{|f(x)|: x \in V_{i}, f \in \Phi\right\} \\
& \leq \sum_{i=1}^{n} \varepsilon+M_{i}=M .
\end{aligned}
$$

Seja agora $D=\{\lambda \in \mathbb{C}:|\lambda| \leq M\}$ e associe a cada $f \in \Phi$ o seguinte ponto $p(f) \in D^{n} \subset \mathbb{C}^{n}$ :

$$
p(f)=\left(f\left(x_{1}\right), \cdots, f\left(x_{n}\right)\right) .
$$

Para cada $f \in \Phi$ e $\varepsilon>0$, defina também $B_{\varepsilon}(f)=B_{\varepsilon}\left(f\left(x_{1}\right)\right) \times \cdots \times B_{\varepsilon}\left(f\left(x_{n}\right)\right)$, com $\left|B_{\varepsilon}(f)\right|<\varepsilon$, onde $B_{\varepsilon}\left(f\left(x_{i}\right)\right)$ é uma bola em $D$ com raio $\varepsilon$ pequeno.

Como $D$ é compacto, $D^{n}=D \times \cdots \times D$ também é compacto. Note que $\cup_{f \in \Phi} B_{\varepsilon}(f) \subset D^{n}$. Além disso, por construção,

$$
D^{n} \backslash \bigcup_{f \in \Phi} B_{\varepsilon}(f)
$$

também é compacto. Logo existe uma cobertura $\mathcal{C}$ de subconjuntos de diâmetro menor que $\varepsilon$, tal que

$$
\mathcal{C} \cup\left(\bigcup_{f \in \Phi} B_{\varepsilon}(f)\right) \supset D^{n} .
$$

Assim, $D^{n}$ possui uma cobertura finita $\mathcal{C}_{1}, \cdots, \mathcal{C}_{m}, B_{\varepsilon}\left(f_{1}\right), \cdots, B_{\varepsilon}\left(f_{m}\right)$ tais que, para toda $f \in$ $\Phi$, existe $k, 1 \leq k \leq m$, de modo que

$$
\left|f\left(x_{i}\right)-f_{k}\left(x_{i}\right)\right|<\varepsilon, 1 \leq i \leq n .
$$

Como para todo $x \in X, x$ está em algum $V_{i}$, segue que, para este $i$

$$
\left|f(x)-f\left(x_{i}\right)\right|<\varepsilon \text { e }\left|f_{k}(x)-f_{k}\left(x_{i}\right)\right|<\varepsilon .
$$

Assim

$$
\begin{aligned}
\left|f(x)-f_{k}(x)\right| & =\left|f(x)-f\left(x_{i}\right)+f\left(x_{i}\right)-f_{k}\left(x_{i}\right)-f_{k}(x)+f_{k}\left(x_{i}\right)\right| \\
& <\left|f\left(x_{i}\right)-f_{k}\left(x_{i}\right)\right|+\left|f(x)-f\left(x_{i}\right)\right|+\left|f_{k}(x)-f_{k}\left(x_{i}\right)\right| \\
& <3 \varepsilon .
\end{aligned}
$$

$\operatorname{Assim}\left|f(x)-f_{k}(x)\right|<3 \varepsilon$ para todo $x \in X$.

Temos então que as bolas centradas em $f_{1}, \cdots, f_{k}$ de raio $3 \varepsilon$ cobrem $\Phi$. Como $\varepsilon$ é arbitrário, $\Phi$ é totalmente limitado. 


\subsection{O Espectro de um Operador Limitado}

Definição 1.7 Dados E, F espaços de Banach, dizemos que um operador $T: E \longrightarrow F$ é limitado (continuo) se existe constante $c \geq 0$ tal que

$$
\|T u\| \leq c\|u\|, \forall u \in E .
$$

Definição 1.8 Um operador limitado $T \in \mathcal{L}(E, F)$, ou seja, $T: E \longrightarrow F$ contínuo, com $E, F$ espaços de Banach é dito compacto se $T\left(B_{E}\right)$ tem fecho compacto em $F$, ou seja a imagem de $T$ por $B_{E}=\{x \in E,\|x\| \leq 1\}$ tem fecho compacto em $F$.

Definição 1.9 Seja $E$ um espaço de Banach e $T \in \mathcal{L}(E)$ operador limitado. O conjunto resolvente, denotado por $\rho(T)$, é definido por

$$
\rho(T)=\{\lambda \in \mathbb{C} ;(T-\lambda I) \text { é bijetiva de } E \text { em } E\} .
$$

O espectro, denotado por $\sigma(T)$, é o complementar do conjunto resolvente, isto é, $\sigma(T)=\mathbb{C} \backslash \rho(T)$. Um número complexo é dito ser um autovalor de $T$ se

$$
N(T-\lambda I) \neq\{0\}
$$

$N(T-\lambda I)$ é o autoespaço correspondente.

Definição 1.10 Um operador $T^{*}: D\left(T^{*}\right) \subset F^{*} \longrightarrow E^{*}$ é chamado adjunto de $T$ se

$$
\langle T u, v\rangle_{E, E^{*}}=\left\langle u, T^{*} v\right\rangle_{F, F^{*}}, \forall u \in D(T), \forall v \in D\left(T^{*}\right),
$$

onde $E^{*}, F^{*}$ são os espaços duais de $E, F$ e $D(T), D\left(T^{*}\right)$ são os dominios de $T$ e $T^{*}$.

Agora, assumiremos que $E=H$ é um espaço de Hilbert e que $T \in \mathcal{L}(H)$. Identificando $H^{*}$ e $H$, veremos $T^{*}$ como um operador de $H$ nele mesmo.

Definição 1.11 Um operador limitado $T \in \mathcal{L}(H)$ é dito ser autoadjunto se $T^{*}=T$, isto é

$$
\langle T u, v\rangle=\left\langle u, T^{*} v\right\rangle \forall u, v \in H .
$$

Definição 1.12 Seja $T: D(T) \subset H \longrightarrow H$ um operador. Dizemos que $T$ é simétrico se

$$
\langle T u, v\rangle=\langle u, T v\rangle \forall u, v \in D(T) .
$$

Observação: Para operadores limitados, as noções de simétrico e auto-adjunto coincidem.

\subsection{Espaços $L^{p}$}

Aqui vamos recordar os espaços $L^{p}$.

Seja $(\Omega, \mathcal{M}, \mu)$ um espaço de medida, isto é, $\Omega$ é um conjunto e

1. $\mathcal{M}$ é uma $\sigma$-álgebra em $\Omega$, ou seja, $\mathcal{M}$ é uma coleção de subconjuntos de $\Omega$ tais que:

- $\emptyset \in \mathcal{M}$,

- Se $A \in \mathcal{M}$, então $A^{c} \in \mathcal{M}$,

- $\cup_{n=1}^{\infty} A_{n} \in \mathcal{M}$ sempre que $A_{n} \in \mathcal{M}$, para todo $n$.

2. $\mu$ é uma medida, isto é, $\mu: \mathcal{M} \longrightarrow[0, \infty]$ satisfaz

- $\mu(\emptyset)=0$, 
- $\mu\left(\cup_{n=1}^{\infty} A_{n}\right)=\cup_{n=1}^{\infty} \mu\left(A_{n}\right)$ sempre que $\left(A_{n}\right)$ é uma família contável disjunta de membros de $\mathcal{M}$.

3. $\Omega$ é $\sigma$-finito, ou seja, existe uma família contável $\left(\Omega_{n}\right)$ em $\mathcal{M}$ tal que $\Omega=\cup_{n=1}^{\infty} \Omega_{n}$ e $\mu\left(\Omega_{n}\right)<\infty$ para todo $n$.

Os conjuntos $E \in \mathcal{M}$ com a propriedade $\mu(E)=0$ são chamados de conjuntos de medida nula, podemos escrevê-lo como $|E|=0$.

Dizemos que uma propriedade vale q.t.p. (para quase todo ponto $x \in \Omega$ ) ou quase sempre se vale para todo $\Omega$ exceto num conjunto de medida nula.

Chamamos de $L^{1}(\Omega)$ o espaço das funções integráveis de $\Omega$ em $\mathbb{R}$

$$
L^{1}(\Omega)=\left\{f: \Omega \longrightarrow \mathbb{R} ; \int_{\Omega} f(x) d \mu<\infty\right\}, x \in \Omega .
$$

Definição 1.13 Seja $p \in \mathbb{R}$ com $1<p<\infty$. Definimos

$$
L^{p}(\Omega)=\left\{f: \Omega \longrightarrow \mathbb{R} ;\|f\|_{L^{p}(\Omega)}=\left(\int_{\Omega}|f(x)|^{p} d \mu\right)^{\frac{1}{p}}<\infty\right\},
$$

e, se $p=\infty$

$L^{\infty}(\Omega)=\{f: \Omega \longrightarrow \mathbb{R} ; f$ é mensurável e existe uma constante $C$ tal que $|f(x)| \leq C$ q.t.p. em $\Omega\}$.

com

$$
\|f\|_{L^{\infty}(\Omega)}=\inf \{C ;|f(x)| \leq C \text { q.t.p. em } \Omega\} .
$$

Notação: Seja $1<p<\infty$; denotamos por $q$ o expoente conjugado

$$
\frac{1}{p}+\frac{1}{q}=1
$$

Quando $p=1$ tomamos $q=\infty$. Analogamente, quando $p=\infty$, definimos seu conjugado por $q=1$.

Proposição 1.5 (Desigualdade de Hölder) Se $f \in L^{p}(\Omega)$ e $g \in L^{q}(\Omega), p, q \geq 1$ e $1 / p+1 / q=1$ então

$$
\int_{\Omega}|f g| d \mu \leq\|f\|_{p} \cdot\|g\|_{q}
$$

Demonstração: Se $\|f\|_{p} \cdot\|g\|_{q}=0$ então $\|f\|_{p}=0$ ou $\|g\|_{q}=0$ e assim $|f \cdot g|=|f \| g|=0$ e portanto $\int_{\Omega}|f g| d \mu=0$.

Se $\|f\|_{p} \cdot\|g\|_{q} \neq 0$, neste caso, temos que mostrar que

$$
\int_{\Omega}\left|\frac{f}{\|f\|_{p}}\right|\left|\frac{g}{\|g\|_{q}}\right| d \mu \leq 1
$$

Note que, como $\left\|\frac{f}{\|f\|_{p}}\right\|_{p}=\left\|\frac{g}{\|g\|_{q}}\right\|_{q}=1$, temos pela Proposição 1.3 que

$$
\begin{aligned}
\int_{\Omega} \frac{|f|}{\|f\|_{p}} \frac{|g|}{\|g\|_{p}} d \mu \leq \int_{\Omega} \frac{\left(\frac{|f|}{\|f\|_{p}}\right)^{p}}{p}+\frac{\left(\frac{|g|}{\|g\|_{q}}\right)^{q}}{q} d \mu & =\frac{1}{p} \int_{\Omega} \frac{|f|^{p}}{\|f\|_{p}^{p}} d x+\frac{1}{q} \int_{\Omega} \frac{|g|^{q}}{\|g\|_{q}^{q}} d \mu \\
& =\frac{1}{p}+\frac{1}{q}=1 .
\end{aligned}
$$

Note que a demonstração vale para o caso $p=\infty$ e $q=1$ onde teremos $0=\lim _{p \rightarrow \infty} \frac{1}{p}$.

Assim, concluímos a prova. 
Proposição 1.6 (Desigualdade de Minkowski) Se $f, g \in L^{p}(\Omega), 1 \leq p \leq \infty$ então

$$
\|f+g\|_{p} \leq\|f\|_{p}+\|g\|_{p}
$$

Demonstração: Se $p=\infty$,

$$
|f+g| \leq|f|+|g| \leq\|f\|_{\infty}+\|g\|_{\infty} .
$$

Mostraremos para $p=1$ e $p>1$.

Se $p=1$, a afirmação é clara já que $\int_{\Omega}|f+g| d \mu \leq \int_{\Omega}|f| d x+\int_{\Omega}|g| d \mu$.

Se $p>1$, notamos que

$$
|f+g|^{p}=|f+g|^{p-1}|f+g| \leq|f||f+g|^{p-1}+|g||f+g|^{p-1} .
$$

Mostremos que $(f+g)^{p-1} \in L^{q}(\Omega)$.

De fato, como $\frac{1}{p}+\frac{1}{q}=1$ então $p q=p+q$, assim

$$
\int_{\Omega}\left(|f+g|^{p-1}\right)^{q} d \mu=\int_{\Omega}|f+g|^{p} d \mu<\infty \Rightarrow(f+g)^{p-1} \in L^{q}(\Omega) .
$$

Daí, temos pela Desigualdade de Hölder

$$
\begin{aligned}
\int_{\Omega}|f+g|^{p} d \mu & \leq \int_{\Omega}|f||f+g|^{p-1} d \mu+\int_{\Omega}|g||f+g|^{p-1} d \mu \\
& \leq\|f\|_{p}\left\|(f+g)^{p-1}\right\|_{q}+\|g\|_{p}\left\|(f+g)^{p-1}\right\|_{q} \\
& =\left(\|f\|_{p}+\|g\|_{p}\right)\left\|(f+g)^{p-1}\right\|_{q} .
\end{aligned}
$$

Então

$$
\frac{\|f+g\|^{p}}{\left\|(f+g)^{p-1}\right\|_{q}} \leq\|f\|_{p}+\|g\|_{p}
$$

Mas,

$$
\left\|(f+g)^{p-1}\right\|_{q}=\left(\int_{\Omega}\left(|f+g|^{p-1}\right)^{q} d \mu\right)^{\frac{1}{q}}=\|f+g\|_{p}^{\frac{p}{q}} .
$$

Daí, de (1.6), obtemos

$$
\|f+g\|_{p}^{p-\frac{p}{q}} \leq\|f\|_{p}+\|g\|_{q} \Rightarrow\|f+g\|_{p} \leq\|f\|_{q}+\|g\|_{p} .
$$

Teorema 1.7 (Fischer-Riesz) O espaço $L^{p}$ é uma espaço de Banach para todo $1 \leq p \leq \infty$.

Demonstração: Faremos os casos $p=\infty$ e $1 \leq p<\infty$.

- Caso $p=\infty$ : Seja $\left\{f_{n}\right\}$ uma sequência de Cauchy em $L^{\infty}(\Omega)$. Dado um inteiro $k \geq 1$, existe um inteiro $N_{k}$ tal que

$$
\left\|f_{m}-f_{n}\right\|_{L^{\infty}(\Omega)} \leq \frac{1}{k}, m, n \geq N_{k} .
$$

Assim, existe um conjunto de medida nula $E_{k}$ tal que

$$
\left|f_{m}-f_{n}\right| \leq \frac{1}{k}, \forall x \in \Omega \backslash E_{k}, \forall m, n \geq N_{k},
$$

pela afirmação.

Então temos $E=\cup_{k} E_{k}$, assim, $E$ tem medida nula, e temos para todo $x \in \Omega \backslash E_{k}$, a sequência $f_{n}$ é de Cauchy (em $\mathbb{R}$ ) e converge para alguma $f$. Desde modo $f_{n}(x) \rightarrow f(x)$ para todo $x \in \Omega \backslash E$. Tomando o limite em (1.7) com $m \rightarrow \infty$, obtemos 


$$
\left|f(x)-f_{n}(x)\right| \leq \frac{1}{k}, \forall x \in \Omega \backslash E, \forall n \geq N_{k} .
$$

Conluímos que $f \in L^{\infty}(\Omega)$ e $\left\|f-f_{n}\right\|_{L^{\infty}(\Omega)} \leq \frac{1}{k}$ para todo $n \geq N_{k}$. Desde modo $f_{n} \rightarrow f$ em $L^{\infty}(\Omega)$.

- Caso $1 \leq p<\infty$ : Seja $\left\{f_{n}\right\}$ uma sequência de Cauchy em $L^{p}(\Omega)$. É suficiente mostrar que uma subsequência converge em $L^{p}(\Omega)$.

Tomemos uma subsequência $\left\{f_{n_{k}}\right\}$ tal que

$$
\left\|f_{n_{k+1}}-f_{n_{k}}\right\| \leq \frac{1}{2^{k}} \cdot \forall k \geq 1
$$

Mostremos que $f_{n_{k}}$ converge em $L^{p}(\Omega)$.

Para simplificar a notação, escrevemos $f_{k}$ ao invés de $f_{n_{k}}$, assim

$$
\left\|f_{k+1}-f_{k}\right\|_{L^{p}(\Omega)} \leq \frac{1}{2^{k}}, \forall k \geq 1
$$

Seja

$$
g_{n}(x)=\sum_{k=1}^{n}\left|f_{k+1}(x)-f_{k}(x)\right|
$$

Note que $\left\|g_{n}\right\|_{L^{p}(\Omega)} \leq 1$.

Como $g_{1} \leq g_{2} \leq \cdots$ q.t.p em $\Omega$ e $\sup _{n} \int_{\Omega} g_{n}<\infty$, segue do Teorema da Convergência Monótona (veja por exemplo [2], Teorema 4.1) que $g_{n}(x)$ tende a uma função $g(x)$ q.t.p em $\Omega$, com $g \in L^{p}(\Omega)$.

Por outro lado, para $m, n \geq 2, m>n$, temos, pela desigualdade triangular

$$
\begin{aligned}
\left|f_{m}(x)-f_{n}(x)\right| & \leq\left|f_{m}(x)-f_{m-1}(x)\right|+\cdots+\left|f_{n+1}(x)-f_{n}(x)\right| \\
& \leq g(x)-g_{n-1}(x) .
\end{aligned}
$$

Segue que $f_{n}(x)$ é de Cauchy, q.t.p em $\Omega$ e converge para um limite finito, $f(x)$.

Temos

$$
\left|f(x)-f_{n}(x)\right| \leq g(x)
$$

q.t.p para $n \geq 2$, e, em particular, $f \in L^{p}(\Omega)$. Finalmente, pelo Teorema da Convergência Dominada (veja [2], Teorema 4.2), segue que $\left\|f_{n}-f\right\| \rightarrow 0$, já que $\left|f_{n}(x)-f(x)\right|^{p} \rightarrow 0$ q.t.p e também $\left|f_{n}(x)-f(x)\right|^{p} \leq g^{p}(x) \in L^{1}(\Omega)$. 


\section{Capítulo 2}

\section{Contração Com Parâmetros e o Teorema de Stampacchia}

Na primeira seção deste capítulo, baseando-se em [5], enunciaremos e demonstraremos o Teorema do Ponto Fixo de Banach assim como o Teorema da Contração com Parâmetros. A partir dele, obtemos o Teorema da Função Implícita. Já na segunda seção, seguiremos [2]. Vamos apresentar a Lei do Paralelogramo e resultados sobre projeções em conjuntos convexos e fechados, para em seguida obtermos o Teorema da Representação de Riesz, que é de grande importância em Análise Funcional e no estudo de EDP's. Em seguida enunciaremos e demonstraremos o Teorema de Stampacchia e seu famoso corolário conhecido como Teorema de Lax-Milgram.

\subsection{Teorema da Contração Uniforme}

Inicialmente mostramos o Teorema do Ponto Fixo em espaços métricos completos

Teorema 2.1 (Banach) Seja $(S, d)$ um espaço métrico completo e seja $T: S \longrightarrow S$ uma contração, isto é, existe $\theta<1$ tal que

$$
d(T(x), T(y)) \leq \theta d(x, y), \forall x \in S .
$$

Então, existe um único ponto fixo de $T$ em $S, a \in S, T(a)=a$. Além disso, para todo $x \in S, a$ sequência $\left(T^{n} a\right)_{n=0}^{\infty}$, onde $T^{n}=\underbrace{T \circ T \circ \cdots \circ T}_{n}$ converge $e$

$$
\lim _{n \rightarrow \infty} T^{(n)}(x)=a
$$

\section{Demonstração:}

Unicidade: Sejam $a$ e $a_{1}$ dois pontos fixos. Temos então

$$
d\left(a, a_{1}\right)=d\left(T(a), T\left(a_{1}\right)\right) \leq \theta d\left(a, a_{1}\right) .
$$

Logo, $d\left(a, a_{1}\right)=0$, portanto $a=a_{1}$.

Existência: Sejam $x \in S$ e $x_{n}=T^{n}(x)$. Mostremos que $\left\{x_{n}\right\}$ é de Cauchy.

Afirmação:

$$
d\left(T^{n}(x), T^{n}(y)\right) \leq \theta^{n} d(x, y) .
$$

De fato, mostremos por indução: Para $n=1$, temos:

$$
d(T(x), T(y)) \leq \theta d(x, y),
$$

o que é verdade, por hipótese. 
Vamos supor válido para $n$ e mostremos que vale para $n+1$. Logo

$$
\begin{aligned}
d\left(T^{n}(T(x)), T^{n}(T(y))\right) \leq & \theta^{n} d(T(x), T(y)) \\
& \leq \theta^{n+1} d(x, y) .
\end{aligned}
$$

Portanto

$$
d\left(T^{n+1}(x), T^{n+1}(y)\right) \leq \theta^{n+1} d(x, y) .
$$

Agora, note que $d\left(x_{n+r}, x_{n}\right) \leq \theta^{n} d\left(x_{r}, x\right)$, e, pela desingualdade triangular, obtemos:

$$
\begin{aligned}
d\left(x_{r}, x\right)=d\left(x, x_{r}\right) & \leq d(x, T(x))+d\left(T(x), T^{2}(x)\right)+\cdots+d\left(T^{r-1}(x), T^{r}(x)\right) \\
& \leq\left(1+\theta+\theta^{2}+\cdots+\theta^{r-1}\right) d(x, T(x)) .
\end{aligned}
$$

Temos então que $\theta$ forma uma progressão geométrica, e assim,

$$
d\left(x, x_{r}\right) \leq \frac{1}{1-\theta} d(x, T(x)) .
$$

Segue por (2.2) e (2.1), que

$$
d\left(x_{n+r}, x_{n}\right) \leq \frac{\theta^{n}}{1-\theta} d(x, y) .
$$

Seja $m=n+r$. Então, dado $\varepsilon>0$, tomemos $n_{0} \in \mathbb{N}$ de tal modo que

$$
n_{0}>\ln \left(\frac{\varepsilon(1-\theta)}{d(x, T(x))}\right) \frac{1}{\ln \theta} .
$$

Concluímos que

$$
d\left(x_{m}, x_{n}\right)<\varepsilon .
$$

Portanto $\left\{x_{n}\right\}$ é de Cauchy e existe $a \in S, S$ é espaço métrico completo, tal que $x_{n} \rightarrow a$.

Falta mostrar que $\lim x_{n}=a$ é ponto fixo de $T(x)$. De fato:

$$
T(a)=T\left(\lim x_{n}\right)=\lim T\left(x_{n}\right)=\lim x_{n+1}=a .
$$

Definição 2.1 Se $(S, d)$ e $(\Lambda, l)$ são espaços métricos completos, dizemos que $T: S \times \Lambda \longrightarrow S$ é uma contração uniforme se existe $\theta<1$ tal que

$$
d(T(x, \lambda), T(y, \lambda)) \leq \theta d(x, y) .
$$

para todo $x, y$ em $S$ e $\lambda$ em $\Lambda$.

Proposição 2.2 Se A é um operador em um espaço de Banach qualquer tal que $\|A\|<1$ e I é a identidade, então $(I-A)$ é inversivel.

Demonstração: Notemos que, se $\|A\|<1$, a série $\sum_{n}^{\infty}\|A\|^{n}$ converge. Sendo assim, dado $\varepsilon>0$, existe $N$ tal que a sequência $\left\{\sum_{n=0}^{m} A^{n}\right\}$ é de Cauchy. De fato, tomando $k, j \in \mathbb{N}$ tal que $k>j$ e $m=l+h$, temos

$$
\begin{aligned}
\left\|\sum_{k=0}^{m} A^{k}-\sum_{j=0}^{l} A^{j}\right\| & =\left\|I+A+\cdots+A^{m}-\left(I+A+\cdots+A^{l}\right)\right\| \\
& =\left\|\sum_{n=l+1}^{m} A^{n}\right\| \leq \sum_{n=l+1}^{m}\|A\|^{n},
\end{aligned}
$$


como $\sum_{n=0}^{\infty}\|A\|^{n}$ é convergente, temos

$$
\sum_{n=l+1}^{m}\left\|A^{n}\right\| \leq \varepsilon
$$

para $l$ suficientemente grande. Logo, segue que $\left\{\sum_{k=0}^{m} A^{k}\right\}$ é de Cauchy.

Agora, mostremos que

$$
\sum_{n=0}^{\infty} A^{n}=(I-A)^{-1}
$$

De fato,

$$
(I-A)\left(I+A+\cdots+A^{m}\right)=I-A^{m+1},
$$

e como $\left\|A^{k}\right\| \leq\|A\|^{k}$ e $\|A\|^{k} \rightarrow 0$ quando $k \rightarrow \infty$ temos que $A^{k} \rightarrow 0$, assim

$$
\lim _{n \rightarrow \infty}(I-A) \underbrace{\left(I+A+\cdots+A^{n}\right)}_{\sum_{n=1}^{m} A^{n}}=\lim _{n \rightarrow \infty}\left(I-A^{n+1}\right)=I .
$$

Segue então que $(I-A)$ é inversível.

Agora vamos provar o Teorema da Contração Uniforme, que é o principal resultado deste Capítulo.

Teorema 2.3 (Teorema da Contração Uniforme) Sejam U,V abertos nos espaços de Banach $X, Y$, e seja $\bar{U}$ o fecho de $U$ (um espaço métrico completo). Suponha $T: \bar{U} \times V \longrightarrow \bar{U}$ contração uniforme em $\bar{U}$, e seja $g(y)$ o único ponto fixo de $T(\cdot, y)$ para cada $y \in V$.

Se $T \in C^{k}(\bar{U} \times V, X), 0 \leq k<\infty$, isto é, a derivada parcial de Frechèt (Definição 1.4) até a ordem $k$ existe em $U \times V$ e se estende continuamente até $\bar{U} \times V$, então a aplicação $y \longmapsto g(y)$ está em $C^{k}(V, X)$.

Se a aplicação $(x, y) \longmapsto T(x, y) \in X$ é analitica em $U_{1} \times V$, onde $U_{1}$ é uma vizinhança de $\bar{U}$, então a aplicação $y \longmapsto g(y)$ é analítica de $V$ em $X$.

Demonstração: Dividiremos a demonstração em dois casos:

- Caso $k=0$

Temos por hipótese que $T$ é contínua e temos que mostrar que $g$ é contínua. Bom, pela continuidade de $T$, dado $\varepsilon_{1}$, existe $\delta>0$ tal que, se $\left\|(x, y)-\left(x_{0}, y_{0}\right)\right\|<\delta$ então $\| T(x, y)-$ $T\left(x_{0}, y_{0}\right) \|<\varepsilon_{1}$.

Agora, como $g$ é ponto fixo, pela desigualdade triangular temos

$$
\begin{aligned}
\left\|g\left(y_{1}\right)-g\left(y_{2}\right)\right\| & =\| T\left(g\left(\left(y_{1}\right), y_{1}\right)-T\left(g\left(y_{2}\right), y_{2}\right) \|\right. \\
& =\| T\left(g\left(\left(y_{1}\right), y_{1}\right)-T\left(g\left(\left(y_{2}\right), y_{1}\right)+T\left(g\left(\left(y_{2}\right), y_{1}\right)-T\left(g\left(\left(y_{2}\right), y_{2}\right) \|\right.\right.\right.\right. \\
& \leq \theta\left\|g\left(y_{1}\right)-g\left(y_{2}\right)\right\|+\varepsilon_{1} .
\end{aligned}
$$

Então

$$
\begin{aligned}
\left\|g\left(y_{1}\right)-g\left(y_{2}\right)\right\|(1-\theta) & \leq \varepsilon_{1} \\
\left\|g\left(y_{1}\right)-g\left(y_{2}\right)\right\| & \leq \frac{\varepsilon_{1}}{1-\theta} .
\end{aligned}
$$

Tomando

$$
\varepsilon=\frac{\varepsilon_{1}}{1-\theta},
$$

segue o resultado. 
- Caso $k=1$

Como $T$ é contração uniforme $\left\|T\left(x_{1}, y\right)-T\left(x_{2}, y\right)\right\| \leq\|\theta\|\left\|x_{1}-x_{2}\right\|$, segue que a derivada com respeito à primeira coordenada, $T_{x}(x, y)$ é tal que $\left\|T_{x}(x, y)\right\| \leq \theta<1$.

Agora, como $g(y)=T(g(y), y)$, encontramos, pela regra da cadeia que $g^{\prime}(y)$ deve ser a solução $M$ da equação:

$$
\begin{gathered}
M=T_{x}(g(y), y) M+T_{y}(g(y), y) \\
\left(I-T_{x}(g(y), y)\right) M=T_{y}(g(y), y) .
\end{gathered}
$$

Mas, $\left\|T_{x}(g(y), y)\right\| \leq \theta<1$, e, pela Proposição 2.2 podemos escrever

$$
M=\left(I-T_{x}(g(y), y)\right)^{-1} T_{y}(g(y), y) .
$$

Note que tal equação possui solução contínua única, $M(y)$. Queremos mostrar que $g^{\prime}(y)=$ $M(y), \operatorname{assim}$

$$
\lim _{\eta \rightarrow 0}\left\|\frac{g(y+\eta)-g(y)-M(y) \eta}{\eta}\right\|=0 .
$$

Para isto, considere $\gamma=g(y+\eta)-g(y)$. Então, usando o fato de $g(y)$ ser ponto fixo para cada $y$, temos

$$
T(g(y)+\gamma, y+\eta)-T(g(y), y)=\gamma,
$$

pois

$$
T(g(y)+g(y+\eta)-g(y), y+\eta)-T(g(y), y)=T(g(y+\eta), y+\eta)-T(g(y), y)=\gamma
$$

Então

$$
\left(I-T_{x}(g(y), y)\right) \gamma=T_{y}(g(y), y) \eta+\Delta,
$$

com $\Delta=T(g(y)+\gamma, y+\eta)-T(g(y), y)-T_{x}(g(y), y) \gamma-T_{y}(g(y), y) \eta$.

Como $T$ é de classe $C^{1}$, segue que

$$
\frac{\left\|T(g(y)+\gamma, y+\eta)-T(g(y), y)-T_{x}(g(y), y) \gamma-T_{y}(g(y), y) \eta\right\|}{\|\gamma\|+\|\eta\|} \rightarrow 0
$$

se $\|\gamma\|+\|\eta\| \rightarrow 0$.

De fato, se $\eta \rightarrow 0$, então $\gamma \rightarrow 0$, já que $g$ é contínua. Portanto, para algum $\varepsilon>0$, com $\|\eta\|$ pequeno o suficiente

$$
\|\Delta(\eta)\|<\varepsilon(\|\gamma(\eta)\|+\|\eta\|) .
$$

Então

$$
\gamma=T_{y}(g(y), y) \eta+\Delta+T_{x}(g(y), y) \gamma
$$

e

$$
\|\gamma\| \leq\left\|T_{y}(g(y), y) \eta\right\|+\|\Delta\|+\theta\|\gamma\| .
$$

Usando (2.4), segue para $\eta$ suficientemente pequeno que 


$$
\begin{aligned}
\|\gamma(\eta)\|\left(1-\frac{\varepsilon}{1-\theta}\right) & \leq \frac{1}{1-\theta}\left\|T_{y}\right\|\|\eta\|+\frac{\varepsilon}{1-\theta}\|\eta\| \\
& \leq \frac{\|\eta\|}{1-\theta}\left(\left\|T_{y}\right\|+\|\varepsilon\|\right) .
\end{aligned}
$$

Tomando $\varepsilon$ pequeno o suficiente, $\|\gamma(\eta)\|=O(\|\eta\|) \operatorname{com} \eta \rightarrow 0$.

Agora, por (2.3),

$$
\Delta(\eta, \gamma(\eta))=\left(I-T_{x}\right)(\gamma(\eta)-M \eta)
$$

Então, encontramos

$$
\begin{aligned}
\|\gamma-M \eta\| & =\left\|\left(I-T_{x}\right)^{-1} \Delta\right\| \\
& \leq\left\|\left(I-T_{x}\right)^{-1}\right\|\|\Delta\| .
\end{aligned}
$$

Portanto,

$$
\|\gamma-M \eta\|=o\|\eta\|
$$

Mas, pela definição de $\gamma$

$$
\|g(y+\eta)-g(y)-M \eta\|=o(\|\eta\|)
$$

Portanto

$$
M(y)=g^{\prime}(y)
$$

- Caso $k>1$

Para este caso, procedemos por indução. De fato, se o resultado vale para $(k-1)$, então quando $T \in C^{k}$, temos por hipótese que $g \in C^{k-1}$, pelo menos, e vale

$$
\left(I-T_{x}(g(y), y)\right) g^{\prime}(y)=T_{y}(g(y), y)
$$

o que nos dá $g^{\prime} \in C^{k-1}, \operatorname{logo} g \in C^{k}$.

Mostremos agora que a aplicação $g(y)$ é analítica, quando, por hipótese, $T: \bar{U} \times V \longrightarrow \bar{U}$ é contração uniforme e analítica em $U_{1} \times V$, com $U_{1}$ aberto de $\bar{U}$.

Tome $(g(y), y) \in U_{1} \times V$. Note que, como $T$ é analítica em $U_{1} \times V \subset X \times Y$ podemos escrever

$$
T(x+\Delta x, y+\Delta y)=T(x, y)+D T(x, y)(\Delta x, \Delta y)+\cdots,
$$

como uma série de Taylor para algum raio de convergência $\rho$.

Vamos então estender $T$ para o plano complexo. Para isto, seja $X_{\mathbb{C}}=\{\bar{x}=u+i v, u, v \in X\}$. Vamos definir a extensão da série $T$ em $X_{\mathbb{C}}$ como

$$
T_{\mathbb{C}}(\bar{x}+\Delta \bar{x}, \bar{y}+\Delta \bar{y})=T(\bar{x}, \bar{y})+D T(\bar{x}, \bar{y})(\Delta \bar{x}, \Delta \bar{y})+\cdots,
$$

Tomando a bola $B(g(y), \rho) \subset X_{\mathbb{C}}$, temos $T_{\mathbb{C}}$ possui o mesmo raio de convergência $\rho$, e $\left.T_{\mathbb{C}}\right|_{X}=T$. Temos então que $T_{\mathbb{C}}$ é analítica, contração uniforme e vale $T_{\mathbb{C}}(g(y), y)=g(y)$. 
Sendo assim, basta mostrarmos que $g$ é $C^{1}$. De fato, isto é mostrado de maneira inteiramente análoga ao que foi feito anteriormente. Então, temos que $g$ é analítica.

Como consequência do Teorema da Contração Uniforme obtemos o Teorema da Função Implícita

Teorema 2.4 (Teorema da Função Implícita) Sejam $X, Y, Z$ espaços de Banach e $U, V$ abertos em $X, V$ respectivamente. Assuma $F: U \times V \longrightarrow Z$ continua e diferenciável, que $\left(x_{0}, y_{0}\right) \in U$, $F\left(x_{0}, y_{0}\right)=0$, e a derivada parcial $F_{x}\left(x_{0}, y_{0}\right) \in \mathcal{L}(X, Y)$ tem inversa contínua.

Então, existe uma vizinhança de $\left(x_{0}, y_{0}\right), U_{1} \times V_{1} \subset U \times V$, e uma função $f: V_{1} \longrightarrow U_{1}, f\left(y_{0}\right)=$ $x_{0}$, tal que para $(x, y) \in U_{1} \times V_{1}, F(x, y)=0$ se, e somente se, $f(y)=x$. Portanto $F(f(y), y)=0$ para todo $y \in V_{1}$.

Se $F: U \times V \longrightarrow Z$ é $C^{k}(1 \leq k<\infty)$ ou analítica perto de $\left(x_{0}, y_{0}\right)$, então $f$ é $C^{k}$ ou analítica, respectivamente, perto de $y_{0}$.

Demonstração: Vamos definir $L=\left(F_{x}\left(x_{0}, y_{0}\right)\right)^{-1} \in \mathcal{L}(Z, X)$ e $G(x, y)=x-L F(x, y)$.

Temos que $G$ é $C^{1}$ numa vizinhança de $\left(x_{0}, y_{0}\right)$ em $X$ (ou $C^{k}$ ou analítica) com $G\left(x_{0}, y_{0}\right)=x_{0}$. Além disso

$$
G_{x}(x, y)=1-\left(F_{x}\left(x_{0}, y_{0}\right)^{-1}\left(F_{x}(x, y)\right),\right.
$$

o que nos dá $G_{x}\left(x_{0}, y_{0}\right)=1-1=0$.

Note que, por continuidade numa vizinhança de $\left(x_{0}, y_{0}\right),\left\|G_{x}(x, y)\right\| \leq \theta<1$, o que nos mostra que $G$ é contração, assim

$$
\left\|G\left(x_{1}, y\right)-G\left(x_{2}, y\right)\right\| \leq \theta\left\|x_{1}-x_{2}\right\| .
$$

Então, para todo $y \in V_{1}$, existe $f(y)$ tal que $G(f(y), y)=f(y)$.

Por outro lado, $f(y)=f(y)-L F(f(y), y)$. Portanto $F(f(y), y)=0$.

A outra afirmação segue diretamente do Teorema 2.3.

\subsection{Teorema de Stampacchia e Lax-Milgram}

Definição 2.2 Denotamos por $E^{*}$ o espaço dual do espaço vetorial normado num corpo $\mathbb{K}=\mathbb{R} e$ E como sendo o espaço de todos os funcionais lineares de $E$ em $\mathbb{K}$, com a norma

$$
\|f\|_{E^{*}}=\sup _{\|x\|<1}|f(x)|=\sup _{\|x\| \leq 1}|f(x)| .
$$

Definição 2.3 Seja E um espaço vetorial normado em um corpo $\mathbb{K}$. Um conjunto $C \subset E$ é dito convexo se

$$
t x+(1-t) y \in C
$$

sempre que $x, y \in C$ e para todo $t \in[0,1]$.

Proposição 2.5 (Lei do Paralelogramo) Seja $H$ um espaço de Hilbert. Para quaisquer $x, y \in$ $H$,

$$
\|x+y\|^{2}+\|x-y\|^{2}=2\left(\|x\|^{2}+\|y\|^{2}\right) .
$$

Demonstração: De fato, $\forall x, y \in H$

$$
\|x+y\|^{2}=\langle x, y\rangle=\langle x, x\rangle+2\langle x, y\rangle+\langle y, y\rangle,
$$

e

$$
\|x-y\|^{2}=\langle x, x\rangle-2\langle x, y\rangle+\langle y, y\rangle .
$$


Assim

$$
\begin{aligned}
\|x+y\|^{2}+\|x-y\|^{2} & =\langle x, x\rangle+2\langle x, y\rangle+\langle y, y\rangle+ \\
& +\langle x, x\rangle-2\langle x, y\rangle+\langle y, y\rangle \\
& =2\|x\|^{2}+2\|y\|^{2} .
\end{aligned}
$$

Observação Podemos escrever a Lei do Paralelogramo da seguinte maneira

$$
\left\|\frac{x+y}{2}\right\|^{2}+\left\|\frac{x-y}{2}\right\|^{2}=\frac{1}{2}\left(\|x\|^{2}+\|y\|^{2}\right),
$$

e sua demonstração é análoga.

Teorema 2.6 (Projeção em um Conjunto Convexo e Fechado) Seja $K \subset H$ um conjunto convexo, fechado e não vazio. Então, para todo $f \in H$ existe um único $u \in K$ tal que

$$
\|f-u\|=\min _{v \in K}\|f-v\|=\operatorname{dist}(f, K) .
$$

Ainda mais, u é caracterizada pela propriedade

$$
u \in K e\langle f-u, v-u\rangle \leq 0 \forall v \in K .
$$

Notação: O elemento $u$ é chamado de projeção de $f$ em $K$, e é denotado por

$$
u=P_{K} f
$$

\section{Demonstração:}

- Existência

Seja $\left\{v_{n}\right\}$ uma sequência de minimização para a equação (2.6), isto é, $v_{n} \in K$ e

$$
d_{n}=\left\|f-v_{n}\right\| \rightarrow d=\inf _{v \in K}\|f-v\| .
$$

Afirmação: $\left\{v_{n}\right\}$ é sequência de Cauchy.

De fato, aplicando a Proposição $2.5 \operatorname{com} x=f-v_{n}$ e $y=f-v_{m}$ nos dá

$$
\left\|\frac{f-v_{n}+f-v_{m}}{2}\right\|^{2}+\left\|\frac{f-v_{n}-\left(f-v_{m}\right)}{2}\right\|^{2}=\frac{1}{2}\left(\left\|f-v_{n}\right\|^{2}+\left\|f-v_{m}\right\|^{2}\right)
$$

ou seja

$$
\left\|f-\frac{v_{n}+v_{m}}{2}\right\|^{2}+\left\|\frac{v_{n}-v_{m}}{2}\right\|^{2}=\frac{1}{2}\left(d_{n}^{2}+d_{m}^{2}\right) .
$$

Como $K$ é convexo, temos que $\frac{v_{n}+v_{m}}{2}$ pertence a $K$, e assim

$$
\left\|f-\frac{v_{n}+v_{m}}{2}\right\| \geq d .
$$

Segue então

$$
\left\|\frac{v_{n}-v_{m}}{2}\right\|^{2} \leq \frac{1}{2}\left(d_{n}^{2}+d_{m}^{2}\right)-d^{2}
$$

e assim

$$
\lim _{m, n \rightarrow \infty}\left\|\frac{v_{n}-v_{m}}{2}\right\|=0
$$


Logo a sequência é de Cauchy, como afirmamos.

Assim, a sequência $\left\{v_{n}\right\}$ converge para algum limite $u \in K \operatorname{com} d=\|f-u\|$.

- Equivalência entre (2.6) e (2.7)

Assuma que $u$ satisfaça (2.6) e seja $w \in K$. Temos que, como $K$ é convexo,

$$
v=(1-t) u+t w \in K \forall t \in[0,1] .
$$

Assim

$$
\|f-u\| \leq\|f-[(1-t) u+t w]\|=\|f-u-t(w-u)\| .
$$

Portanto

$$
\begin{aligned}
\|f-u\|^{2} & \leq\|f-u-t(w-u)\|^{2}=\langle f-u-t(w-u), f-u-t(w-u)\rangle \\
& \leq\|f-u\|^{2}-2 t\langle f-u, w-u\rangle+t^{2}\|w-u\|^{2} .
\end{aligned}
$$

Logo, para qualquer $t \in(0,1]$

$$
2\langle f-u, w-u\rangle \leq t\|w-u\|^{2}
$$

Fazendo $t \rightarrow 0^{+}$obtemos

$$
\langle f-u, w-u\rangle \leq 0
$$

o que nos dá (2.7).

Por outro lado, assumindo que $u$ satisfaz (2.7), temos

$$
\|u-f\|^{2}-\|v-f\|^{2}=2\langle f-u, v-u\rangle-\|u-v\|^{2} \leq 0 \forall v \in K,
$$

já que

$$
\begin{aligned}
\langle f-u, v-u\rangle & =\langle f-u, f-u+v-f\rangle \\
& =\|f-u\|^{2}+\langle f-u, v-f\rangle \\
& =\|f-u\|^{2}+\langle f-v-u+v, v-f\rangle \\
& =\|f-u\|^{2}-\|v-f\|^{2}+\langle v-u, v-f\rangle \\
& =\|f-u\|^{2}-\|v-f\|^{2}+\|v-u\|^{2}-\langle v-u, f-u\rangle,
\end{aligned}
$$

e o resultado segue.

- Unicidade

Assuma que $u_{1}$ e $u_{2}$ satisfaça (2.7). Temos

$$
\left\langle f-u_{1}, v-u_{1}\right\rangle \leq 0, \forall v \in K
$$

$\mathrm{e}$

$$
\left\langle f-u_{2}, v-u_{2}\right\rangle \leq 0, \forall v \in K .
$$

Escolhendo $v=u_{2}$ em (2.8) e $v=u_{1}$ em (2.9) e somando, encontramos

$$
\begin{aligned}
\left\langle f-u_{1}, u_{2}-u_{1}\right\rangle+\left\langle f-u_{2}, u_{1}-u_{2}\right\rangle & =\left\langle f, u_{2}-u_{1}\right\rangle-\left\langle u_{1}, u_{2}-u_{1}\right\rangle \\
& +\left\langle f, u_{1}-u_{2}\right\rangle-\left\langle u_{2}, u_{1}-u_{2}\right\rangle \\
& =\left\langle u_{1}, u_{1}\right\rangle-\left\langle u_{1}, u_{2}\right\rangle-\left\langle u_{2}, u_{1}\right\rangle+\left\langle u_{2}, u_{2}\right\rangle \\
& \leq 0 .
\end{aligned}
$$


Donde tiramos

$$
\left\langle u_{1}-u_{2}, u_{1}-u_{2}\right\rangle \leq 0,
$$

ou seja

$$
\left\langle u_{1}-u_{2}, u_{1}-u_{2}\right\rangle=0
$$

Logo $u_{1}=u_{2}$.

Proposição 2.7 Seja $K \subset H$ um conjunto convexo fechado e não vazio. Então $P_{K}$ não aumenta distância, isto é

$$
\left\|P_{K} f_{1}-P_{K} f_{2}\right\| \leq\left\|f_{1}-f_{2}\right\| \forall f_{1}, f_{2} \in H .
$$

Demonstração: Tome $u_{1}=P_{K} f_{1}$ e $u_{2}=P_{K} f_{2}$. Temos

$$
\left\langle f_{1}-u_{1}, v-u_{1}\right\rangle \leq 0 \forall v \in K
$$

e

$$
\left\langle f_{2}-u_{2}, v-u_{2}\right\rangle \leq 0 \forall v \in K .
$$

Tomando $v=u_{2}$ em 2.10 e $v=u_{1}$ em 2.9 e somando, obtemos

$$
\begin{aligned}
\left\langle f_{1}-u_{1}, u_{2}-u_{1}\right\rangle+\left\langle f_{2}-u_{2}, u_{1}-u_{2}\right\rangle & \leq 0 \\
-\left\langle f_{1}-u_{1}, u_{1}-u_{2}\right\rangle+\left\langle f_{2}-u_{2}, u_{1}-u_{2}\right\rangle & \leq 0 \\
\left\langle f_{2}-u_{2}+u_{1}-f_{1}, u_{1}-u_{2}\right\rangle & \leq 0 \\
\left\langle u_{1}-u_{2}, u_{1}-u_{2}\right\rangle & \leq \mid\left\langle f_{1}-f_{2}, u_{1}-u_{2}\right\rangle \\
\left\|u_{1}-u_{2}\right\|^{2} & \leq\left\|f_{1}-f_{2}\right\|\left\|u_{1}-u_{2}\right\| .
\end{aligned}
$$

Segue então que

$$
\left\|u_{1}-u_{2}\right\| \leq\left\|f_{1}-f_{2}\right\|
$$

Corolário 2.8 Assuma que $M \subset H$ é um subespaço fechado linear. Seja $f \in H$, então $u=P_{M} f$ é caracterizado por

$$
u \in M e\langle f-u, v\rangle=0 \forall v \in M .
$$

Além disso, $P_{M}$ é um operador linear, chamado de projeção ortogonal.

Demonstração: Por (2.7), temos

$$
\langle f-u, v-u\rangle \leq 0 \forall v \in M
$$

e assim

$$
\langle f-u, t v-u\rangle \leq 0, \forall t \in \mathbb{R},
$$

pois, como $M$ é subespaço linear, segue então que

$$
t\left\langle f-u, v-\frac{u}{t}\right\rangle \leq 0 .
$$

Analisaremos alguns casos:

- Se $t>0$, tomando $t \rightarrow \infty$, temos $\langle f-u, v\rangle \leq 0$.

- Se $t<0$, tomando $t \rightarrow-\infty$, temos $\langle f-u, v\rangle \geq 0$. 
Portanto, $\langle f-u, v\rangle=0$. Segue que $(2.12)$ é válida.

Por outro lado, se $u$ satisfaz (2.12),

$$
\langle f-u, v-u\rangle=0,
$$

pois $v-u \in M, \forall v \in M$.

Falta mostrar que $P_{M} f$ é linear.

- $P_{M} f_{1}+P M f_{2}=P_{M}\left(f_{1}+f_{2}\right)$

De fato, sejam $P_{M} f_{1}=u_{1}$ e $P_{M} f_{2}=u_{2}$. Então, $\forall v \in M$ temos

$$
\begin{aligned}
& \left\langle f_{1}-u_{1}, v\right\rangle=0 \\
& \left\langle f_{2}-u_{2}, v\right\rangle=0
\end{aligned}
$$

$\mathrm{e}$

$$
\left\langle\left(f_{1}+f_{2}\right)-\tilde{u}, v\right\rangle=0 .
$$

Subtraindo as equações acima, encontramos

$$
\left\langle\left(f_{1}+f_{2}\right)-\tilde{u}-f_{1}-f_{2}+\left(u_{1}+u_{2}\right), v\right\rangle=0, \forall v \in M
$$

ou seja

$$
\left\langle\left(u_{1}+u_{2}\right)-\tilde{u}, v\right\rangle=0, \forall v \in M
$$

O que implica

$$
\left\|u_{1}+u_{2}-\tilde{u}\right\|^{2}=0 \forall v \in M
$$

E assim

$$
P_{M}\left(f_{1}+f_{2}\right)=\tilde{u}=u_{1}+u_{2} .
$$

- $P_{M} \lambda f=\lambda P_{M} f$

De fato, dados $f \in H$ e $\lambda \in \mathbb{R}$ e se $P_{M} f=u$ e $P_{M} \lambda f=w$ então

$$
\begin{aligned}
\langle f-u, v\rangle & =0 \forall v \in M \\
\langle\lambda f-w, v\rangle & =0 \forall v \in M .
\end{aligned}
$$

Agora, considerando $\langle\lambda f-\lambda u, v\rangle=0$ e subtraindo de $\langle\lambda f-w, v\rangle=0$, encontramos

$$
\begin{aligned}
\langle\lambda f-\lambda f-(w-\lambda u), v\rangle & =0, \forall v \in M \\
\langle-w+\lambda u, v\rangle & =0, \forall v \in M .
\end{aligned}
$$

O que implica

$$
\|\lambda u-w\|^{2}=0 .
$$

E assim

$$
w=\lambda u .
$$

Teorema 2.9 (Teorema da Representação de Riesz) Seja $H$ um espaço de Hilbert em $\mathbb{R}$ com produto interno $\langle.,$.$\rangle e \varphi \in H^{*}$. Então existe um único $f \in H$ tal que

$$
\varphi(x)=\langle x, f\rangle, \forall x \in H .
$$

Além disso, $\|\varphi\|_{H^{*}}=\|f\|$. 
Demonstração: Seja $M=N(\varphi)=\{u \in H: \varphi(u)=0\}=\varphi^{-1}\{0\}$. Note que $M \subset H$ é fechado, pois $\{0\}$ é fechado em $\mathbb{R}$ e como $\varphi$ é contínua, sua imagem inversa mapeia fechados em fechados.

Vamos assumir $M \neq H$, pois caso contrário $\varphi \equiv 0$ e a conclusão do Teorema é óbvia, bastando tomar $f=0$.

Mostremos que existe $g \in H$ tal que $\|g\|=1$ e $\langle g, v\rangle=0, \forall v \in M$, e assim $g \notin M$.

De fato, tome $g_{0} \in H$ com $g_{0} \notin M$. Seja $g_{1}=P_{M} g_{0}$. Então

$$
g=\frac{g_{0}-g_{1}}{\left\|g_{0}-g_{1}\right\|}
$$

é tal que $\|g\|=1$ e, pelo Corolário $2.8,\langle g, v\rangle=0$.

Agora, dado qualquer $u \in H$, tome $v=u-\lambda g, \operatorname{com} \lambda=\frac{\varphi(u)}{\varphi(g)}$. que

Note que $v$ está bem definida, pois $\varphi(g)=\frac{1}{\left\|g_{0}-g_{1}\right\|}\left[\varphi\left(g_{0}\right)-\varphi\left(g_{1}\right)\right] \neq 0$. Ainda mais, $v \in M$, já

$$
\varphi(v)=\varphi(u-\lambda g)=\varphi(u)-\lambda \varphi(g)=0 .
$$

Segue que $\langle g, v\rangle=0$, isto é,

$$
\begin{aligned}
0=\langle g, v\rangle=\langle g, u-\lambda g\rangle & =\langle g, u\rangle-\lambda\langle g, g\rangle \\
& =\varphi(g)\langle g, u\rangle-\varphi(g) \frac{\varphi(u)}{\varphi(g)}\|g\|^{2}
\end{aligned}
$$

Donde concluímos

$$
\begin{aligned}
0 & =\varphi(g)\langle g, u\rangle-\varphi(u) \\
\varphi(u) & =\varphi(g)\langle g, u\rangle, \forall u \in H .
\end{aligned}
$$

Tomando $f=\varphi(g) g$, o resultado segue.

Falta mostrar que $\|\varphi\|_{H^{*}}=\|f\|$.

De fato,

$$
\|\varphi\|=\sup _{u \in H\|u\|=1}|\varphi(u)|=\sup _{u \in H\|u\|=1}|\langle f, u\rangle|=\|f\| .
$$

Definição 2.4 Seja $V$ um espaço vetorial sobre $\mathbb{R}$. Uma forma bilinear é uma função $B: V \times V \longrightarrow$ $\mathbb{R}$ que é linear em ambas as variáveis, ou seja, para todo $u, v \in V$ temos

$$
\begin{aligned}
B\left(u+u^{\prime}, v\right) & =B(u, v)+B\left(u^{\prime}, v\right), \\
B\left(u, v+v^{\prime}\right) & =B(u, v)+B\left(u, v^{\prime}\right), \\
B(\lambda u, v) & =B(u, \lambda v)=\lambda B(u, v) .
\end{aligned}
$$

Definição 2.5 Uma forma bilinear $a: H \times H \longrightarrow \mathbb{R}$ é dita ser

- contínua, se existe uma constante c tal que

$$
\|a(u, v)\| \leq c\|u\|\|v\|, \forall u, v \in H
$$

- coerciva, se existe uma constante $\alpha>0$ tal que

$$
a(v, v) \geq \alpha\|v\|^{2}, \forall v \in H .
$$

Definição 2.6 Duas normas $\|\cdot\|_{1}$ e $\|\cdot\|_{2}$ sobre um espaço vetorial E são ditas equivalentes se existem constantes $c_{1}$ e $c_{2}$ com $c_{1} \leq c_{2}$ tais que

$$
c_{1}\|x\|_{1} \leq\|x\|_{2} \leq c_{2}\|x\|_{1}, \forall x \in E .
$$


Teorema 2.10 (Stampacchia) Suponhamos que a(.,.) seja uma forma bilinear contínua e coerciva no espaço de Hilbert $H$. Seja $K \subset H$ um subconjunto convexo, fechado e não vazio. Então, dado qualquer $\varphi \in H^{*}$, existe um único elemento $u \in K$ tal que

$$
a(u, v-u) \geq \varphi(v-u), \forall v \in K .
$$

Além disso, se $a(.,$.$) for simétrica, ou seja a(u, v)=a(v, u), \forall u, v \in H$, então u pode ser caracterizado pela seguinte propriedade:

$$
u \in K
$$

$e$

$$
\frac{1}{2} a(u, u)-\varphi(u)=\min _{v \in K}\left\{\frac{1}{2} a(v, v)-\varphi(v)\right\}
$$

Demonstração: Sabemos do Teorema 2.9 que existe um único $f \in H$ tal que

$$
\varphi(v)=\langle f, v\rangle, \forall v \in H .
$$

Por outro lado, se fixarmos $u \in H$, a aplicação $v \longmapsto a(u, v)$ é um funcional linear contínuo em $H$. Usando o Teorema 2.9 novamente, encontramos um único elemento em $H$, denotado por $A u$, tal que

$$
a(u, v)=\langle A u, v\rangle, \forall v \in H .
$$

Temos que $A$ é linear. Além disso, $A$ é contínuo. De fato, temos que existe $\varphi_{u} \in H^{*}$ tal que, para todo $v \in H$

$$
\varphi_{u}(v)=a(u, v)=\langle A u, v\rangle .
$$

Segue que,

$$
\|A u\|=\left\|\varphi_{u}\right\|=\sup _{\|v\| \leq 1}|a(u, v)| \leq \sup _{\|v\| \leq 1} c\|u\|\|v\| \leq c\|u\|
$$

e

$$
a(u, u)=\langle A u, u\rangle \geq \alpha\|u\|^{2} .
$$

Assim, o problema (2.13) é equivalente ao problema

$$
\langle A u, v-u\rangle \geq\langle f, v-u\rangle, \forall v \in K,
$$

ou seja, queremos demonstrar que

$$
\langle f-A u, v-u\rangle \leq 0, \forall v \in K .
$$

Dado $\rho>0$, consideremos o problema

$$
\langle\rho f-\rho A u, v-u\rangle \leq 0, \forall v \in K,
$$

ou ainda,

$$
\langle\rho f-\rho A u+u-u, v-u\rangle \leq 0, \forall v \in K .
$$

Assim, queremos encontrar $u \in K$ tal que $u=P_{K}(\rho f-\rho A u+u)$.

Definamos $S: K \longrightarrow K$ por $S(w)=P_{K}(\rho f-\rho A w+w)$. Pela Proposição 2.7, temos

$$
\left\|S\left(w_{1}\right)-S\left(w_{2}\right)\right\| \leq\left\|\left(w_{1}-w_{2}\right)-\rho\left(A w_{1}-A w_{2}\right)\right\| .
$$


Assim,

$$
\begin{aligned}
\left\|S\left(w_{1}\right)-S\left(w_{2}\right)\right\|^{2} & \leq\left\|\left(w_{1}-w_{2}\right)\right\|^{2}-2 \rho\left\langle A w_{1}-A w_{2}, w_{1}-w_{2}\right\rangle+\rho^{2}\left\|A w_{1}-A w_{2}\right\|^{2} \\
& \leq\left\|\left(w_{1}-w_{2}\right)\right\|^{2}-2 \rho \alpha\left\|\left(w_{1}-w_{2}\right)\right\|^{2}+\rho^{2} c^{2}\left\|\left(w_{1}-w_{2}\right)\right\|^{2} \\
& \leq\left\|\left(w_{1}-w_{2}\right)\right\|^{2}\left(1-2 \rho \alpha+\rho^{2} c^{2}\right) .
\end{aligned}
$$

Escolhendo $\rho>0$ e $c>\alpha>0$ de modo que

$$
k^{2}=1-2 \alpha \rho+\rho^{2} c^{2}<1,
$$

quando $\rho=\frac{\alpha}{c^{2}}, k^{2}$ é mínimo e vale $1-\frac{\alpha^{2}}{c^{2}}$, concluímos que $S$ é uma contração em $K$. Segue pelo Teorema 2.1 que $S(u)=u$, ou seja

$$
\begin{aligned}
\rho f-\rho A u+u & =u \\
A u & =f,
\end{aligned}
$$

o que mostra (2.13).

Suponhamos agora que a forma bilinear a seja simétrica. Assim $a(.,$.$) define um novo produto$ interno em $H$ e uma nova norma $\|u\|_{1}=(a(u, u))^{\frac{1}{2}}$.

De fato, note que

- $a(u, v)=a(v, u)$ por hipótese;

- $a(u+v, w)=a(u, w)+a(v, w)$, pois $a$ é forma bilinear;

- $a(\lambda u, v)=\lambda a(u, v)$;

- $a(u, u)>0$ e igual a zero se, e somente se $u=0$, pois $a$ é coerciva.

Note que esta nova norma é equivalente à primeira com constantes de equivalência $c$ e $\alpha$.

Segue que $\left(H,\|\cdot\|_{1}\right)$ é um espaço de Hilbert. Pelo Teorema 2.9, para cada $\varphi \in H^{*}$, existe um único $g \in H$ tal que

$$
\varphi(v)=a(g, v), \forall v \in H
$$

Assim, queremos resolver

$$
a(g-u, v-u) \leq 0, \forall v \in K .
$$

A solução de (2.15) é a projeção sobre $K$ (relativa ao produto interno $a(.,$.$) ).$

Pelo Teorema 2.6, sabemos que $u$ é o único elemento de $K$ que atinge

$$
\min _{v \in K} a(g-v, g-v)^{\frac{1}{2}} .
$$

Assim, $u$ minimiza a função

$$
\begin{aligned}
v \longmapsto a(g-v, g-v) & =a(v, v)-2 a(g, v)+a(g, g) \\
& =a(v, v)-2 \varphi(v)+a(g, g) .
\end{aligned}
$$

Concluímos que $u$ satisfaz

$$
\frac{1}{2} a(u, u)-\varphi(u) \leq \frac{1}{2} a(v, v)-\varphi(v), \forall v \in K .
$$

Corolário 2.11 (Lax-Milgram) Suponhamos a $(\cdot, \cdot)$ seja uma forma bilinear contínua e coerciva no espaço de Hilbert $H$. Então dado qualquer $\varphi \in H^{*}$, existe um único elemento $u \in H$ tal que

$$
a(u, v)=\varphi(v), \forall v \in H .
$$


Além disso, se a $(\cdot, \cdot)$ for simétrica, então u pode ser caracterizado pela seguinte propriedade:

$$
u \in H
$$

$e$

$$
\frac{1}{2} a(u, u)-\varphi(u)=\min _{u \in H}\left\{\frac{1}{2} a(v, v)-\varphi(v)\right\} .
$$

Demonstração: Tome $K=H$ no Teorema 2.10 e use um argumento análogo ao que foi usado na prova do Corolário 2.8 .

Agora finalizamos o capítulo com o seguinte resultado sobre o espectro de operadores autoadjuntos.

Teorema 2.12 Seja $T \in \mathcal{L}(H)$ um operador autoadjunto. Defina

$$
m=\inf _{u \in H|u|=1}\langle T(u),(u)\rangle \text { e } M=\sup _{u \in H|u|=1}\langle T(u),(u)\rangle .
$$

Então $\sigma(T) \subset[m, M], m \in \sigma(T)$ e $M \in \sigma(T)$. Ainda mais,

$$
\|T\|=\max \{|m|,|M|\} .
$$

Demonstração: Seja $\lambda>M$. Provemos que $\lambda \in \rho(T)$. Note que, para $u \neq 0$

$$
\langle T(u),(u)\rangle=\|u\|^{2}\left\langle T\left(\frac{u}{\|u\|}\right),\left(\frac{u}{\|u\|}\right)\right\rangle \leq M\|u\|^{2} .
$$

Por outro lado

$$
-\langle T(u)-\lambda u, u\rangle \leq\|T(u)-\lambda u\|\|u\|
$$

e também

$$
\begin{aligned}
-\langle T(u)-\lambda u, u\rangle & =-\langle T(u), u\rangle+\lambda\|u\|^{2} \\
& \geq-\|u\|^{2} M+\lambda\|u\|^{2} \\
& =\alpha\|u\|^{2} .
\end{aligned}
$$

Assim

$$
\|T(u)-\lambda u\| \geq \alpha\|u\| .
$$

Logo, se tomarmos $a(u, v)=\langle T(u)-\lambda u, v\rangle$, temos que $a$ é coerciva.

Agora,

$$
\begin{aligned}
|a(u, v)| & =|\langle T(u)-\lambda u, v\rangle| \\
& \leq\|T(u)-\lambda u\|\|v\| \\
& \leq\|T-\lambda\|\|u\|\|v\| \\
& \leq c\|u\|\|v\| .
\end{aligned}
$$

Segue então que $a$ é contínua.

Pelo Corolário 2.11, dado $\varphi \in H^{*}$, existe um único $u \in H$ tal que

$$
\langle T(u)-\lambda u, v\rangle=\varphi(v) .
$$

Pelo Teorema 2.9, concluímos que

$$
\langle T(u)-\lambda u, v\rangle=\langle f, v\rangle,
$$

para algum $f \in H$.

Portanto $(T-\lambda I)$ é bijeção, e assim $\lambda \in \rho(T)$. 
Agora, seja $\lambda<m$. Para $u \neq 0$, escrevemos

$$
\langle T(u),(u)\rangle=\|u\|^{2}\left\langle T\left(\frac{u}{\|u\|}\right),\left(\frac{u}{\|u\|}\right)\right\rangle \geq m\|u\|^{2} .
$$

Logo,

$$
\langle T(u)-\lambda u, u\rangle \leq\|T(u)-\lambda u\|\|u\|
$$

e

$$
\begin{aligned}
\langle T(u)-\lambda u, u\rangle & =\langle T(u), u\rangle-\lambda\|u\|^{2} \\
& \geq(m-\lambda)\|u\|^{2} \\
& =\delta\|u\|^{2} .
\end{aligned}
$$

De modo análogo, $a(u, v)=\langle T(u)-\lambda u, v\rangle$ é contínua e coerciva, então, pelo Corolário 2.11, segue que $\lambda \in \rho(T)$.

Provemos agora que $M \in \sigma(T)(m \in \sigma(T)$ é análogo).

A forma $a(u, v)=\langle M(u)-T(u), v\rangle$ é simétrica e satisfaz

$$
\langle M u-T(u), v\rangle=\langle M(v), v\rangle-\langle T(v), v\rangle \geq 0, \forall v \in H,
$$

pois $M=\sup _{u \in H}\langle T(u), u\rangle$ e $T$ é auto-adjunto.

Assim, satisfaz a desigualdade de Cauchy-Schwarz

$$
|a(u, v)| \leq a(u, u)^{\frac{1}{2}} a(v, v)^{\frac{1}{2}}, \forall u, v \in H,
$$

isto é,

$$
|\langle M(u)-T(u), v\rangle| \leq\langle M(u)-T(u), u\rangle^{\frac{1}{2}}\langle M(v)-T(v), v\rangle^{\frac{1}{2}}, \forall u, v \in H,
$$

Segue que

$$
\|M(u)-T(u)\| \leq c\langle M(u)-T(u), u\rangle^{\frac{1}{2}}, \forall u \in H .
$$

Pela definição de $M$, existe uma sequência $\left\{u_{n}\right\}$ tal que $\left|u_{n}\right|=1$ e $\left\langle T\left(u_{n}\right), u_{n}\right\rangle \rightarrow M$.

De (2.16), temos que

$$
\begin{aligned}
\left\|M\left(u_{n}\right)-T\left(u_{n}\right)\right\| & \leq c\left\langle M\left(u_{n}\right)-T\left(u_{n}\right), u_{n}\right\rangle^{\frac{1}{2}} \\
& \leq c\left[M\left\langle u_{n}, u_{n}\right\rangle-\left\langle T\left(u_{n}\right), u_{n}\right\rangle\right]^{\frac{1}{2}} \rightarrow 0 .
\end{aligned}
$$

Finalmente, mostremos que $\|T\|=\mu$, onde $\mu=\max \{|m|,|M|\}$. De fato, escrevemos, para todo $u, v \in H$, como $T$ é auto-adjunto

$$
\begin{aligned}
& \langle T(u+v), u+v\rangle=\langle T(u), u\rangle+\langle T(v), v\rangle+2\langle T(u), v\rangle \\
& \langle T(u-v), u-v\rangle=\langle T(u), u\rangle+\langle T(v), v\rangle-2\langle T(u), v\rangle .
\end{aligned}
$$

Subtraindo as equações, temos

$$
\langle T(u+v), u+v\rangle-\langle T(u-v), u-v\rangle=4\langle T(u), v\rangle .
$$

Então

$$
\begin{aligned}
4|\langle T(u), v\rangle| & =|\langle T(u+v), u+v\rangle-\langle T(u-v), u-v\rangle| \\
& \leq|\langle T(u+v), u+v\rangle|+|\langle T(u-v), u-v\rangle| \\
& \leq \mu\left(\|u+v\|^{2}+\|u-v\|^{2}\right) .
\end{aligned}
$$

Pela Proposição 2.5,

$$
4|\langle T(u), v\rangle| \leq 2 \mu\left(\|u\|^{2}+\|v\|^{2}\right)
$$


Substituindo $v$ por $\alpha v$, com $\alpha>0$, segue que

$$
\begin{aligned}
4|\langle T(u), \alpha v\rangle| & \leq 2 \mu\left(\|u\|^{2}+\|\alpha v\|^{2}\right) \\
4 \alpha|\langle T(u), v\rangle| & \leq 2 \mu\left(\|u\|^{2}+\alpha^{2}\|v\|^{2}\right) \\
4|\langle T(u), v\rangle| & \leq 2 \mu\left(\frac{\|u\|^{2}}{\alpha}+\alpha\|v\|^{2}\right) .
\end{aligned}
$$

Agora, tomando $\alpha=\frac{\|u\|}{\|v\|}$, obtemos

$$
|\langle T(u), v\rangle| \leq \mu\|u\|\|v\|, \forall u, v \in H .
$$

Então $\|T\| \leq \mu$.

Por outro lado, $|\langle T(u), v\rangle| \leq\|T\|\|u\|^{2}$, pois $M=\|T\|$.

Temos então que $|m| \leq\|T\|$ e $M \leq\|T\|$ e $\operatorname{assim} \mu \leq\|T\|$.

Logo, $\|T\|=\mu$. 


\section{Capítulo 3}

\section{Problema de Neumann Não Local}

Baseando-se em $[1,10]$, estudaremos neste capítulo o problema de Neumann não local. Mostraremos a existência e unicidade de soluções utilizando o Teorema do Ponto Fixo de Banach 2.1 e também estudaremos o comportamento assintótico das soluções com relação à variável temporal $t$. Consideraremos o caso linear e semilinear com linearidades globalmente Lipschitz. Na última seção, estudaremos a regularidade das soluções com relação a alguns parâmetros do nosso problema. Para tanto, utilizaremos o Teorema da Contração Uniforme 2.3.

\subsection{Equação Linear Não Homogênea}

Nesta seção vamos estudar questões de existência e unicidade da seguinte equação de difusão não local linear e não homogênea

$$
\left\{\begin{array}{l}
u_{t}(t, x)=\int_{\Omega} J(x-y)(u(t, y)-u(t, x)) d y+f(t, x), \\
u(0, x)=u_{0}(x)
\end{array}\right.
$$

onde $x \in \Omega \subset \mathbb{R}^{N}$ é um domínio aberto limitado e conexo, $t \in \mathbb{R}$, a condição inicial $u_{0} \in L^{2}(\Omega)$ e $f \in C\left(\mathbb{R}, L^{2}(\Omega)\right)$, com

(H) $J \in C\left(\mathbb{R}^{N}, \mathbb{R}\right)$, não negativa, com $J(0)>0, J(-x)=J(x)$ para todo $x \in \mathbb{R}^{N} \mathrm{e}$

$$
\int_{\mathbb{R}^{N}} J(x) d x=1 .
$$

Também assumimos, sem perda de generalidade que $\|J\|_{L^{\infty}\left(\mathbb{R}^{N}\right)}<\infty$.

A seguir obtemos alguns resultados preliminares necessários para a prova dos resultados principais.

\subsubsection{Resultados Preliminares}

A próxima proposição é um resultado útil que utilizaremos mais a frente.

Proposição 3.1 Seja J satisfazendo $(\boldsymbol{H})$, então para $u \in L^{p}(\Omega)$ e $v \in L^{q}(\Omega)$, com $\frac{1}{p}+\frac{1}{q}=1$, vale

$$
\int_{\Omega} v(x) \int_{\Omega} J(x-y)(u(y)-u(x)) d y d x=-\frac{1}{2} \int_{\Omega} \int_{\Omega} J(x-y)(u(y)-u(x))(v(y)-v(x)) d y d x .
$$


Em particular, se $p=2$, temos, para $u \in L^{2}(\Omega)$

$$
\int_{\Omega} u(x) \int_{\Omega} J(x-y)(u(y)-u(x)) d y d x=-\frac{1}{2} \int_{\Omega} \int_{\Omega} J(x-y)(u(y)-u(x))^{2} d y d x .
$$

Demonstração: Chamando a integral do lado direito de (3.2) de

$$
\begin{aligned}
I_{1} & =\int_{\Omega} \int_{\Omega} J(x-y)(u(y)-u(x))(v(y)-v(x)) d y d x \\
& =\int_{\Omega} \int_{\Omega} J(x-y)(u(y)-u(x)) v(y) d y d x-\int_{\Omega} \int_{\Omega} J(x-y)(u(y)-u(x)) v(x) d y d x .
\end{aligned}
$$

Trocando a ordem das variáveis no primeiro termo da soma, obtemos

$$
I_{1}=\int_{\Omega} \int_{\Omega} J(y-x)(u(x)-u(y)) v(x) d x d y-\int_{\Omega} \int_{\Omega} J(x-y)(u(y)-u(x)) v(x) d y d x .
$$

Como $J(x)=J(-x)$, segue

$$
I_{1}=\int_{\Omega} \int_{\Omega} J(x-y)\left(u(x)-u(y) v(x) d x d y-\int_{\Omega} \int_{\Omega} J(x-y)(u(y)-u(x)) v(x) d y d x .\right.
$$

Graças ao Teorema de Fubini, temos

$$
I_{1}=-2 \int_{\Omega} \int_{\Omega} J(x-y)(u(y)-u(x)) v(x) d y d x .
$$

Assim sendo, provamos que o lado direito de (3.2) é

$$
\begin{array}{r}
\int_{\Omega} \int_{\Omega} J(x-y)(u(y)-u(x)(v(y)-v(x) d y d x= \\
=-2 \int_{\Omega} \int_{\Omega} J(x-y)(u(y)-u(x)) v(x) d y d x .
\end{array}
$$

Por outro lado

$$
\begin{array}{r}
\int_{\Omega} v(x) \int_{\Omega} J(x-y)(u(y)-u(x) d y d x= \\
=\int_{\Omega}\left(\int_{\Omega} J(x-y) u(y) d y-\int_{\Omega} J(x-y) u(x)\right) v(x) d x \\
=\int_{\Omega} \int_{\Omega} J(x-y)(u(y)-u(x)) v(x) d y d x .
\end{array}
$$

Então, de (3.4) e (3.5), temos

$$
\begin{aligned}
& \int_{\Omega} v(x) \int_{\Omega} J(x-y)(u(y)-u(x)) d y d x= \\
& =\int_{\Omega} \int_{\Omega} J(x-y)(u(y)-u(x)) v(x) d y d x= \\
& =-\frac{1}{2} \int_{\Omega} \int_{\Omega} J(x-y)(u(y)-u(x))(v(y)-v(x)) d y d x .
\end{aligned}
$$

A segunda parte da Proposição é um caso imediato de (3.2).

Inicialmente consideramos a equação não local do tipo Neumann homogênea e linear 


$$
\left\{\begin{array}{l}
u_{t}(t, x)=\int_{\Omega} J(x-y)(u(t, y)-u(t, x)) d y \\
u(0, x)=u_{0}(x)
\end{array}\right.
$$

$\operatorname{com} x \in \Omega, t>0$.

Definição 3.1 Uma solução para o problema (3.1) e (3.6) (quando $f \equiv 0$ ) é uma função u $\in$ $C\left([0, \infty], L^{1}(\Omega)\right)$ tal que

$$
u(t, x)=u_{0}(x)+\int_{0}^{t} \int_{\Omega} J(x-y)(u(s, y)-u(s, x)) d y d s+\int_{0}^{t} f(s, x) d x
$$

onde $x \in \Omega, t>0$.

Observação: Apesar de estarmos definindo as soluções em $L^{1}(\Omega)$, a maioria de nossos resultados serão enunciados em $L^{2}(\Omega){ }^{1}$

Proposição 3.2 Para todo $u_{0} \in L^{1}(\Omega)$ a única solução u de (3.6) preserva a massa total em $\Omega$, ou seja

$$
\int_{\Omega} u(t, x) d x=\int_{\Omega} u_{0}(x) d x, \forall t \geq 0
$$

Demonstração: Como

$$
u(t, x)-u_{0}(x)=\int_{0}^{t} \int_{\Omega} J(x-y)(u(s, y)-u(s, x)) d y d s,
$$

integrando em $x$ e utilizando o Teorema de Fubini, obtemos

$$
\begin{aligned}
\int_{\Omega} u(t, x) d x-\int_{\Omega} u_{0}(x) d x & =\int_{\Omega} \int_{0}^{t} \int_{\Omega} J(x-y)(u(s, y)-u(s, x)) d y d s d x \\
& =\int_{0}^{t} \int_{\Omega} \int_{\Omega} J(x-y)(u(s, y)-u(s, x)) d y d x d s \\
& =\int_{0}^{t} \int_{\Omega}\left[\int_{\Omega} J(x-y) u(s, y) d y-\int_{\Omega} J(x-y) d y u(s, x)\right] d x d s \\
& =\int_{0}^{t}\left[\int_{\Omega} \int_{\Omega} J(x-y) u(s, y) d y d x-\int_{\Omega} J(x-y) d y u(x) d x\right] d s \\
& =0
\end{aligned}
$$

Lema 3.3 A função $A: \Omega \longrightarrow \mathbb{R}$ dada por $A(x)=\int_{\Omega} J(x-y) d y$ é contínua e satisfaz $A(x) \geq c$, para algum $c>0$.

Demonstração: Como $J$ é contínua por $\mathbf{( H )}$, temos que é uniformemente contínua em conjuntos compactos de $\mathbb{R}^{N}$. Logo, dado $\varepsilon>0$, existe $\delta>0$ tal que se $|x-z|<\delta$, então $|J(x-y)-J(z-y)|<\varepsilon$. Agora, note que

$$
\begin{gathered}
|A(x)-A(z)|=\left|\int_{\Omega} J(x-y) d y-\int_{\Omega} J(z-y) d y\right| \\
\quad \leq \int_{\Omega}|J(x-y)-J(z-y)| d y \leq \int_{\Omega} \varepsilon d y=\varepsilon|\Omega| .
\end{gathered}
$$

Desta forma, concluímos que $A$ é contínua em $\Omega$.

\footnotetext{
${ }^{1}$ Note também que $L^{2}(\Omega) \subset L^{1}(\Omega)$.)
} 
Mostremos agora que $A(x)$ é estritamente positiva. De fato, suponha que não. Então existe uma sequência $\left\{x_{n}\right\} \subset \Omega$ tal que $0 \leq A\left(x_{n}\right) \leq \frac{1}{n}$. Segue então que $A\left(x_{n}\right) \rightarrow 0$. Note que $A\left(x_{n}\right) \rightarrow A(x)$ se $x \in \Omega$ pela continuidade de $A$. Portanto

$$
0=A(x)=\int_{\Omega} J(x-y) d y>0,
$$

absurdo, pois, por $\mathbf{( H )}, J(0) \geq c>0$.

Analogamente, se $x \in \partial \Omega$, temos $\int_{\Omega} J(x-y) d y=0$, o que também é absurdo, pois $J(0)>0$ e $J$ é contínua. Segue então que $A$ é estritamente positivo e pode ser estendida continuamente até $\bar{\Omega}$.

Seja $C^{k}(\Omega)$ o espaço das funções contínuas $k$-vezes diferenciáveis em $\Omega$, com $k \geq 0$. Se $g \in C^{k}(\Omega)$ e $\alpha=\left(\alpha_{1}, \cdots, \alpha_{n}\right)$ é um multi-index de tamanho $|\alpha|=\alpha_{1}+\cdots+\alpha_{n}$, com $|\alpha| \leq k$, escrevemos

$$
D^{\alpha} g=\frac{\partial^{\alpha_{1}}}{\partial x_{1}^{\alpha_{1}}} \frac{\partial^{\alpha_{2}}}{\partial x_{2}^{\alpha_{2}}} \cdots \frac{\partial^{\alpha_{n}}}{\partial x_{n}^{\alpha_{n}}} g .
$$

Teorema 3.4 Sejam $f \in L^{1}(\Omega)$ e $g \in C^{k}(\Omega)$, com $D^{\alpha} g$ limitada para todo $|\alpha| \leq k$. Então

$$
\int_{\Omega} g(x-y) f(y) d y \in C^{k}(\Omega)
$$

$e$

$$
D^{\alpha}(f * g)=f * D^{\alpha} g
$$

se $|\alpha| \leq k$.

Ainda mais, as derivadas de ordem menor ou igual a $k$ de $f * g$ são limitadas.

Demonstração: Dividiremos a prova em casos.

- Caso $k=0$. Note que $g(x-y)$ é majorada para alguma constante $C$, que independe de $x$ e $y$, ou seja $|f(y) g(x-y)| \leq C|f(y)|$ e é contínua. Portanto, a integral é finita para cada $x$ e pelo Teorema de Continuidade e Derivação sob o sinal de Integração (consulte obra [9], Capítulo 1 , página 20), temos que $f * g$ é contínua.

- Caso $k=1$. Fixemos $j=1, \cdots, n$. Pelo caso anterior, segue que $f * \partial_{x_{j}} g$ é contínua. Para quaisquer $x$ e $y$, temos

$$
\left|\partial_{x_{j}}[f(y) g(x-y)]\right|=\left|f(y)\left(\partial_{x_{j}} g\right)(x-y)\right| \leq C|f(y)| \in L^{1}(\Omega) .
$$

Então, pelo Teorema de Continuidade e Derivação sob o sinal de Integração, temos que existe $\partial_{x_{j}}(f * g)$ e que $\partial_{x_{j}}(f * g)=f *\left(\partial_{x_{j}} g\right)$ é contínua. Variando $j$, concluímos que $f * g \in C^{1}(\Omega)$.

- Caso $k>1$. Segue por indução de maneira inteiramente análoga ao que foi feito anteriormente.

Agora notemos que vale, pela desigualdade de Hölder

$$
\left\|D^{\alpha}(f * g)\right\|_{L^{\infty}(\Omega)}=\left\|f * D^{\alpha} g\right\|_{L^{\infty}(\Omega)} \leq\|f\|_{L^{1}(\Omega)}\left\|D^{\alpha} g\right\|_{L^{\infty}(\Omega)}<\infty,
$$

se $0 \leq|\alpha|<k$.

Definição 3.2 Uma solução de (3.6) é dita estacionária se for independente do tempo t. Nesse caso, a solução estacionária de (3.6) é descrita por

$$
0=\int_{\Omega} J(x-y)(\varphi(y)-\varphi(x)) d y
$$


Proposição 3.5 Toda solução estacionária da equação (3.6) é constante em $\Omega$, e, como a massa total é preservada, a única solução estacionária com a mesma massa de $u_{0}$ é

$$
\varphi=\frac{1}{|\Omega|} \int_{\Omega} u_{0}
$$

Demonstração: Primeiramente, note que a equação (3.7) e o Lema 3.3 nos diz que

$$
\begin{aligned}
\int_{\Omega} J(x-y) d y \varphi(x) & =\int_{\Omega} J(x-y) \varphi(y) d y \\
A(x) \varphi(x) & =\int_{\Omega} J(x-y) \varphi(y) d y \\
\varphi(x) & =\frac{\int_{\Omega} J(x-y) \varphi(y) d y}{A(x)} .
\end{aligned}
$$

Pelo Teorema 3.4 e pelo Lema 3.3, temos que $\varphi$ é contínua. Com efeito, $\varphi$ pode ser estendida continuamente em $\bar{\Omega}$.

Defina

$$
K=\max _{x \in \bar{\Omega}} \varphi(x)
$$

e considere o conjunto $\mathcal{A}=\{x \in \bar{\Omega}: \varphi(x)=K\}$. Temos que $\mathcal{A}$ é fechado e não vazio, pois $\varphi$ é contínua em $\bar{\Omega}$.

Afirmação $\mathcal{A}$ é aberto em $\bar{\Omega}$. Seja $x_{0} \in \mathcal{A}$, então

$$
0=\int_{\Omega} J\left(x_{0}-y\right)\left(\varphi(y)-\varphi\left(x_{0}\right)\right) d y
$$

e, como $x_{0}$ é ponto de máximo, $\varphi(y) \leq \varphi\left(x_{0}\right)$ o que nos dá que, para todo $y \in \Omega \cap B\left(x_{0}, d\right)$, $\varphi(y)=\varphi\left(x_{0}\right)$, para qualquer $B(0, d) \subset \operatorname{supp}(J)$. Logo, conseguimos uma bola aberta dentro de $\mathcal{A}$, implicando que $\mathcal{A}$ é aberto.

Como $\Omega$ é conexo, $\bar{\Omega}$ é conexo, e então $\mathcal{A}=\bar{\Omega}$. Assim $\varphi$ é constante. Segue da Proposição 3.2 que a solução estacionária preserva a massa

$$
\int_{\Omega} \varphi=\int_{\Omega} u_{0} \Rightarrow \varphi|\Omega|=\int_{\Omega} u_{0} \Rightarrow \varphi=\frac{1}{|\Omega|} \int_{\Omega} u_{0},
$$

onde $u_{0}$ é a condição inicial.

Proposição 3.6 Dada J satisfazendo (H), a quantidade

$$
\lambda_{1}:=\lambda_{1}(J, \Omega)=\inf _{u \in L^{2}(\Omega)} \int_{\Omega} u=0 \frac{\frac{1}{2} \int_{\Omega} \int_{\Omega} J(x-y)(u(y)-u(x))^{2} d y d x}{\int_{\Omega}(u(x))^{2} d x}
$$

é estritamente positiva.

Demonstração: É claro que $\lambda_{1} \geq 0$. Provaremos que $\alpha_{1}$ é de fato estritamente positiva. Para este fim, considere o subespaço $W$ de $L^{2}(\Omega)$ dado pelo complemento ortogonal das constantes, ou seja

$$
H=\left\{u \in L^{2}(\Omega): \int_{\Omega} u=0\right\}
$$

e o operador $T: H \longrightarrow H$ dado por

$$
T(u)(x)=\int_{\Omega} J(x-y)(u(x)-u(y)) d y=-\int_{\Omega} J(x-y) u(y) d y+A(x) u(x),
$$


onde $A(x)=\int_{\Omega} J(x-y) d y$. Temos que $T$ é simétrico, pois

$$
\begin{aligned}
\int_{\Omega} T u(x) v(x) d x & =\int_{\Omega} \int_{\Omega} J(x-y)(u(x)-u(y)) d y v(x) d x \\
& =\frac{1}{2} \int_{\Omega} \int_{\Omega} J(x-y)(u(x)-u(y))(v(x)-v(y)) d y d x \\
& =\int_{\Omega} u(x) T v(x) d x
\end{aligned}
$$

pela Proposição 3.1.

Mostremos que $T$ é a soma de um operador inversível e um operador compacto. Para isto, observamos inicialmente que $J(x-\cdot) \in L^{2}(\Omega)$, para todo $x \in \Omega$. Com efeito, pela Desigualdade de Hölder 1.5, temos

$$
\begin{aligned}
\|J(x-\cdot)\|_{L^{2}(\Omega)}^{2} & =\int_{\Omega}|J(x-y)|^{2} d y \leq\|1\|_{L^{1}(\Omega)}\|J(x-\cdot)\|_{L^{\infty}(\Omega)}^{2} \\
& \leq|\Omega| \sup _{z \in \mathbb{R}^{n}}|J(z)|^{2}<\infty .
\end{aligned}
$$

Agora, seja $S: \bar{\Omega} \longrightarrow L^{2}(\Omega)$ definido por $S(x)=J(x-\cdot)$. Note que $S \in C\left(\bar{\Omega}, L^{2}(\Omega)\right)$, pois, para quaisquer $x, z \in \bar{\Omega}$

$$
\|S(x)-S(z)\|_{L^{2}(\Omega)}^{2}=\int_{\Omega}|J(x-y)-J(z-y)|^{2} d y .
$$

Fixemos um compacto $K$ com a propriedade $\{x-y ; x, y \in \Omega\} \subset K$. Dado $\varepsilon>0$, existe $\delta>0$ tal que se $\left|w-w^{\prime}\right|<\delta$, com $w, w^{\prime} \in K \subset \mathbb{R}^{n}$, então

$$
\left|J(w)-J\left(w^{\prime}\right)\right|<\frac{\sqrt{\varepsilon}}{\sqrt{|\Omega|}},
$$

pois $J$ é uniformemente contínua em qualquer compacto de $\mathbb{R}^{n}$. Agora, note que

$$
\|S(x)-S(z)\|_{L^{2}(\Omega)}^{2} \leq \frac{\varepsilon}{|\Omega|}
$$

se $|x-z|<\delta$, já que $|x-y-(z-y)|=|x-z|$.

Segue da Proposição $1.1 S$ é uniformemente contínua em $\bar{\Omega}$, e portanto uniformemente limitada em $\Omega$.

Afirmação: O conjunto $\Phi=\left\{G u \in L^{2}(\Omega):\|u\|_{L^{2}(\Omega)} \leq 1\right\}$, onde $G: L^{2}(\Omega) \longrightarrow L^{2}(\Omega)$, definido por $G u(x)=\int_{\Omega} J(x-y) u(y) d y$ é totalmente limitado e $G$ é um operador compacto.

De fato, note que $G u: \Omega \longrightarrow \mathbb{R} \in C(\Omega)$. Além disso

$$
|G(u(x))|=\left|\int_{\Omega} J(x-y) u(y) d y\right| \leq\|J(x-\cdot)\|_{L^{2}(\Omega)} \leq\|J\|_{L^{\infty}(\Omega)}|\Omega|^{\frac{1}{2}},
$$

assim

$$
\sup _{x \in \Omega}\{|G(u(x))|: G u \in \Phi\}<\infty .
$$


Se $\varepsilon>0$, para todo $x \in \Omega$ existe $\delta>0$ tal que

$$
\begin{aligned}
|G(u(x))-G(u(z))| & =\left|\int_{\Omega} J(x-y) u(y) d y-\int_{\Omega} J(z-y) u(y) d y\right| \\
& \leq\|J(x-\cdot)-J(z-\cdot)\|_{L^{2}(\Omega)}\|u\|_{L^{2}(\Omega)} \leq\|J(x-\cdot)-J(z-\cdot)\|_{L^{2}(\Omega)} \\
& =\left(\int_{\Omega}|J(x-y)-J(z-y)|^{2} d y\right)^{\frac{1}{2}}, \\
& <\left(\int_{\Omega} \frac{\varepsilon^{2}}{|\Omega|} d y\right)^{\frac{1}{2}}=\varepsilon,
\end{aligned}
$$

sempre que $|x-z|<\delta$. Aqui estamos usando que $J$ é uniformemente contínua em qualquer compacto de $\mathbb{R}^{n}$.

Como tal estimativa independe $u$ com $\|u\|_{L^{2}(\Omega)} \leq 1$, temos para todo $|x-z|<\delta$ e para todo $G u \in \Phi$, a desigualdade acima. Ou seja, $\Phi$ é pontualmente limitado e equicontínuo.

Segue pelo Teorema de Arzelá-Ascoli 1.4 que $\Phi$ é totalmente limitada.

Assim, dado $\varepsilon>0$, existe $n_{\varepsilon} \in \mathbb{N}$ tal que

$$
\Phi \subset \bigcup_{i=1}^{n} B_{\varepsilon}\left(x_{i}\right) .
$$

Logo, existe subsequência $\left\{G u_{n}\right\} \subset \Phi$ de Cauchy. O que nos diz que $G$ é compacto.

Afirmação: O operador $N: H \subset L^{2}(\Omega) \longrightarrow L^{2}(\Omega)$ dado por $N(u(x))=A(x) u(x)$ é um operador inversível.

De fato, do Lema 3.3, temos que $A(x) \geq c>0$ e assim, $B$ dada por

$$
B u(x)=\frac{u(x)}{A(x)}
$$

é inversa de $N$.

Agora, note que $T$ é simétrica e limitada, o que nos diz que é auto-adjunta. E mais, segue pela Proposição 2.12 que seu espectro verifica $\sigma(T) \subset[m, M]$, onde $m=\inf _{u \in H}\|u\|_{L^{2}(\Omega)}=1\langle T u, u\rangle$ e $M=\sup _{u \in H}\|u\|_{L^{2}(\Omega)}=1\langle T u, u\rangle$.

Note que, de modo semelhante ao que foi feito na Proposição 3.1, obtemos

$$
\begin{aligned}
m & =\inf _{u \in H\|u\|_{L^{2}(\Omega)}=1}\langle T u, u\rangle \\
& =\inf _{u \in H\|u\|_{L^{2}(\Omega)}=1} \frac{1}{2} \int_{\Omega} \int_{\Omega} J(x-y)(u(x)-u(y))^{2} d y d x \\
& =\lambda_{1} .
\end{aligned}
$$

Então $m \geq 0$. Mostremos que $m>0$. Suponha por absurdo que $m=0$. Temos que $0 \in \sigma(T)$ e $T u=0$ para algum $u \in H$ e $u \neq 0$, ou seja $N(T) \neq\{0\}$ e $T$ não é injetora. Segue pela Proposição 3.5 que $u$ deve ser constante e igual a zero, já que sua integral é nula. Logo $m>0$ e assim, $\alpha_{1}>0$.

Precisaremos, também, dos seguintes Lemas para mostrar a existência e unicidade do problema (3.1): 
Lema 3.7 Seja $f \in C\left([a, b], L^{2}(\Omega)\right)$. Então a função $g:[a, b] \longrightarrow L^{2}(\Omega)$, definida por

$$
g(t)=\int_{a}^{t} f(s, \cdot) d s
$$

é uniformemente contínua.

Demonstração: Note que $\|g(t)\|_{L^{2}(\Omega)}<\infty$, pois

$$
\begin{aligned}
\|g(t)\|_{L^{2}(\Omega)}^{2}=\int_{\Omega}\left(\int_{a}^{t} 1 . f(s, x) d s\right)^{2} d s & \leq \int_{\Omega}\left[\left(\int_{a}^{t} 1^{2} d s\right)^{\frac{1}{2}}\left(\int_{a}^{t}(f(s, x))^{2}\right)^{\frac{1}{2}}\right]^{2} d x \\
& \leq \int_{\Omega}(t-a) \int_{a}^{t}(f(s, x))^{2} d s d x \\
& \leq(t-a) \int_{\Omega} \int_{a}^{t}(f(s, x))^{2} d s d x .
\end{aligned}
$$

Pelo Teorema de Fubini

$$
\begin{aligned}
\|g(t)\|_{L^{2}(\Omega)}^{2} & \leq(t-a) \int_{a}^{t}\|f(s, x)\|_{L^{2}(\Omega)}^{2} d s \\
& \leq(t-a)^{2} \sup _{s \in[a, b]}\|f(s, \cdot)\|_{L^{2}(\Omega)}^{2} \\
& \leq(t-a)^{2} k<\infty,
\end{aligned}
$$

com $k$ constante. Note que $k$ existe pois $f$ é uma função contínua com domínio compacto, logo admite máximo.

Mostremos agora a continuidade uniforme. Sem perda de generalidade vamos mostrar que, para cada $\varepsilon>0$, existe $\delta>0$, tal que se $\left|t-t_{1}\right|<\delta$ então $\left\|g(t)-g\left(t_{1}\right)\right\|_{L^{2}(\Omega)}<\varepsilon$, supondo $t>t_{1}$, com $t, t_{1} \in[a, b]$.

Temos

$$
\left\|g(t)-g\left(t_{1}\right)\right\|_{L^{2}(\Omega)}^{2}=\int_{\Omega}\left(\int_{t_{1}}^{t} \bar{f}(s, x) d s\right)^{2} d x .
$$

Pela Desigualdade de Hölder 1.5

$$
\begin{aligned}
\left\|g(t)-g\left(t_{1}\right)\right\|_{L^{2}(\Omega)}^{2} & \leq \int_{\Omega}\left[\left(\int_{t_{1}}^{t} 1^{2} d s\right)^{\frac{1}{2}}\left(\int_{t_{1}}^{t}(f(s, x))^{2}\right)^{\frac{1}{2}}\right]^{2} d x \\
& \leq\left|t-t_{1}\right| \int_{\Omega} \int_{t_{1}}^{t}(f(s, x))^{2} d s d x .
\end{aligned}
$$

Pelo Teorema de Fubini

$$
\begin{aligned}
\left\|g(t)-g\left(t_{1}\right)\right\|_{L^{2}(\Omega)}^{2} & \leq\left|t-t_{1}\right| \int_{t_{1}}^{t} \int_{\Omega}(f(s, x))^{2} d x d s \\
& \leq\left|t-t_{1}\right| \int_{t_{1}}^{t}\|f(s, x)\|_{L^{2}(\Omega)}^{2} d s \\
& \leq\left(t-t_{1}\right)^{2} \sup _{s \in[a, b]}\|f(s, x)\|_{L^{2}(\Omega)}^{2} \leq\left(t-t_{1}\right)^{2} k,
\end{aligned}
$$

onde $k$ é uma constante dependente de $f$.

Tomando $\delta=\frac{\varepsilon}{(b-a)^{2} k}$, o resultado segue. 
Lema 3.8 Dados A como no Lema 3.3 e $u_{0} \in L^{2}(\Omega)$ temos que a função $S:[a, b] \longrightarrow L^{2}(\Omega)$ dada por $S(t)=e^{-A(\cdot) t} u_{0}(\cdot)$, com $A(\cdot)=\int_{\Omega} J(\cdot-y) d y$, é uniformemente contínua em $[a, b]$.

Demonstração: Dado $\varepsilon>0$, vamos mostrar que $\exists \delta>0$ tal que se $\left|t-t_{1}\right|<\delta$, então

$$
\left\|e^{-A(\cdot) t} u_{0}(\cdot)-e^{-A(\cdot) t_{1}} u_{0}(\cdot)\right\|_{L^{2}(\Omega)}<\varepsilon
$$

Temos, pela Desigualdade de Hölder 1.5 e tomando o supremo que

$$
\int_{\Omega}\left|u_{0}(x)\left(e^{-A(x) t}-e^{-A(x) t_{1}}\right)\right|^{2} d x \leq\left\|e^{-A(\cdot) t}-e^{-A(\cdot) t_{1}}\right\|_{L^{\infty}(\Omega)}^{2}\left\|u_{0}(\cdot)\right\|_{L^{2}(\Omega)}^{2} .
$$

Note que, como $e^{-A(\cdot) t_{1}} \leq 1$ para todo $t$ positivo,

$$
\left\|e^{-A(\cdot) t_{1}}\left(e^{A(\cdot)\left(t_{1}-t\right)}-1\right)\right\|_{L^{\infty}(\Omega)} \leq\left\|e^{A(\cdot)\left(t_{1}-t\right)}-1\right\|_{L^{\infty}(\Omega)} .
$$

Daí, segue de (3.9) que

$$
\int_{\Omega}\left|u_{0}(x)\left(e^{-A(x) t}-e^{-A(x) t_{1}}\right)\right|^{2} d x \leq\left\|e^{A(\cdot)\left(t_{1}-t\right)}-1\right\|_{L^{\infty}(\Omega)} .
$$

Além disso,

$$
\left|A(\cdot)\left(t_{1}-t\right)\right| \leq\|J\|_{L^{\infty}(\Omega)}|\Omega|\left|t_{1}-t\right| .
$$

Chamando $M=\|J\|_{L^{\infty}(\Omega)}|\Omega|,(3.9)$ fica

$$
\begin{aligned}
\left\|u_{0}\right\|_{L^{2}(\Omega)}\left\|e^{A(\cdot)\left(t_{1}-t\right)}-1\right\|_{L^{\infty}(\Omega)} & =\left\|u_{0}\right\|_{L^{2}(\Omega)}\left\|\sum_{n=1}^{\infty} \frac{\left[A(\cdot)\left(t_{1}-t\right)\right]^{n}}{n !}\right\|_{L^{\infty}(\Omega)} \\
& \leq\left\|u_{0}\right\|_{L^{2}(\Omega)} \sum_{n=1}^{\infty} \frac{\left|M\left(t_{1}-t\right)\right|^{n}}{n !} \leq\left\|u_{0}\right\|_{L^{2}(\Omega)} \sum_{n=1}^{\infty} \frac{(M \delta)^{n}}{n !} \\
=\left\|u_{0}\right\|_{L^{2}(\Omega)}\left(e^{\delta M}-1\right) . &
\end{aligned}
$$

Mas queremos

$$
\left\|u_{0}\right\|_{L^{2}(\Omega)}^{2}\left(e^{\delta M}-1\right)<\varepsilon
$$

isto é,

$$
e^{\delta M}<\frac{\varepsilon}{\left\|u_{0}\right\|_{L^{2}(\Omega)}^{2}}+1
$$

Aplicando o logaritmo natural nos dois lados da desigualdade acima, temos que, se tomarmos

$$
\delta=\frac{1}{M} \ln \left(\frac{\varepsilon}{\left\|u_{0}\right\|_{L^{2}(\Omega)}}+1\right),
$$

o resultado segue.

Corolário 3.9 Dados $A$ como no Lema 3.3 e $u \in L^{2}(\Omega)$, temos que as funções $Q, L:[a, b] \longrightarrow$ $L^{2}(\Omega)$ definidas por $Q(t)=\int_{0}^{t} e^{A(\cdot) s} f(s, \cdot) d s$ e $L(t)=\int_{0}^{t} e^{A(\cdot) s}\left(\int_{\Omega} J(\cdot-y) u(s, y) d y\right) d s$, com $f \in$ $C\left(\mathbb{R}, L^{2}(\Omega)\right)$ são uniformemente continuas. 
Demonstração: É consequência dos Lemas 3.7 e 3.8 já que as funções

$$
\bar{f}_{1}(t, \cdot)=e^{A(\cdot) t} f(t, \cdot) \text { e } \bar{f}_{2}(t, \cdot)=e^{A(\cdot) t} \int_{\Omega} J(\cdot-y) u(t, y) d y
$$

pertencem a $C\left([a, b], L^{2}(\Omega)\right)$.

\subsubsection{Existência e Unicidade}

Agora já estamos em condições de provar o resultado principal desta seção. Consideramos a equação não local linear e homogênea (3.1).

Teorema 3.10 Seja $J \in C\left(\mathbb{R}^{N}, \mathbb{R}\right)$ uma função satisfazendo (H) e $f \in C\left(\mathbb{R}, L^{2}(\Omega)\right)$. Então, para cada $u_{0} \in L^{2}(\Omega)$, o problema não local (3.1) possui uma única solução global

$$
u: \mathbb{R} \times \Omega \longrightarrow \mathbb{R}
$$

tal que, para todo intervalo limitado $[a, b] \subset \mathbb{R}$, temos

$$
u \in C^{1}\left([a, b], L^{2}(\Omega)\right)
$$

com

$$
u(t, x)=e^{-A(x) t} u_{0}+\int_{0}^{t} e^{-A(x)(t-s)} \int_{\Omega} J(x-y) u(s, y) d y d s+\int_{0}^{t} e^{-A(x)(t-s)} f(s, x) d s
$$

em $(t, x) \in \mathbb{R} \times \Omega$, onde $A(x) \in L^{\infty}(\Omega)$ é a função positiva definida anteriormente por

$$
A(x)=\int_{\Omega} J(x-y) d y, x \in \Omega .
$$

Além disso, existem constantes positivas $\alpha$ e $C$, tais que

$$
\left\|u(t, \cdot)-\frac{1}{|\Omega|} \int_{\Omega} u_{0}(x) d x\right\|_{L^{2}(\Omega)} \leq e^{-\alpha t}\left[\left\|u_{0}-\frac{1}{|\Omega|} \int_{\Omega} u_{0}(x) d x\right\|_{L^{2}(\Omega)}+C \int_{0}^{t}\|f(s, \cdot)\|^{2} d s\right] .
$$

Se ainda assumirmos que $u_{0} \in L^{\infty}(\Omega)$,

$$
\int_{0}^{\infty}\|f(s, \cdot)\|_{L^{2}(\Omega)}^{2} d s<\infty
$$

$e$

$$
\int_{0}^{\infty} e^{\gamma s}\|f(s, \cdot)\|_{L^{\infty}}<\infty
$$

para $\gamma=\|J\|_{L^{\infty}\left(\mathbb{R}^{N}\right)}|\Omega|$, então

$$
\left\|u(t, \cdot)-\frac{1}{|\Omega|} \int_{\Omega} u_{0}(x) d x\right\|_{L^{\infty}(\Omega)} \leq C e^{-\alpha t}
$$

para qualquer $t \geq 0$.

Demonstração: Primeiramente, mostremos a equivalência da equação (3.10) e do problema (3.1). Reescrevendo (3.1)

$$
u_{t}(t, x) e^{A(x) t}=e^{A(x) t} \int_{\Omega} J(x-y) u(t, y) d y-e^{A(x) t} \int_{\Omega} J(x-y) d y u(t, x)+e^{A(x) t} f(t, x) .
$$


Como $\left(u(t, x) e^{A(x) t}\right)^{\prime}=u_{t}(t, x) e^{A(x) t}+A(x) u(t, x) e^{A(x) t}$, segue que

$$
\left(u(t, x) e^{A(x) t}\right)^{\prime}=e^{A(x) t} \int_{\Omega} J(x-y) u(t, y) d y+e^{A(x) t} f(t, x) .
$$

Integrando

$$
\begin{aligned}
& u(t, x) e^{A(x) t}-u(0, x)=\int_{0}^{t} e^{A(x) s} \int_{\Omega} J(x-y) u(s, y) d y d s+\int_{0}^{t} e^{A(x) s} f(s, x) d s \\
& u(t, x)=e^{-A(x) t} u(0, x)+\int_{0}^{t} e^{-A(x)(t-s)} \int_{\Omega} J(x-y) u(s, y) d y d s+\int_{0}^{t} e^{-A(x)(t-s)} f(s, x) d s .
\end{aligned}
$$

Agora, partindo de

$$
u(t, x) e^{A(x) t}=u_{0}(x)+\int_{0}^{t} e^{A(x) s} \int_{\Omega} J(x-y) u(s, y) d y d s+\int_{0}^{t} e^{A(x) s} f(s, x) d s
$$

e derivando

$$
\begin{aligned}
u_{t}(t, x) e^{A(x) t}+A(x) u(t, x) e^{A(x) t} & =e^{A(x) t} \int_{\Omega} J(x-y) u(t, y) d y+e^{A(x) t} f(t, x) \\
u_{t}(t, x)+\int_{\Omega} J(x-y) d y u(t, x) & =\int_{\Omega} J(x-y) u(t, y) d y+f(t, x) \\
u_{t}(t, x) & =\int_{\Omega} J(x-y)(u(t, y)-u(t, x)) d y+f(t, y) .
\end{aligned}
$$

Agora, mostremos a existência e unicidade. Consideremos $T>0$ fixo no espaço de Banach

$$
X_{T}=\left\{u \in C\left([-T, T], L^{2}(\Omega)\right): \sup _{-T \leq t \leq T}\|u(t, \cdot)\|_{L^{2}(\Omega)}<\infty\right\} .
$$

A solução será dada como um ponto fixo do operador

$$
F: X_{T} \longrightarrow X_{T}
$$

definido por

$$
\begin{aligned}
F(u)(t, x) & =e^{-A(x) t} u_{0}+\int_{0}^{t} e^{-A(x)(t-s)} \int_{\Omega} J(x-y) u(s, y) d y d s \\
& +\int_{0}^{t} e^{-A(x)(t-s)} f(s, x) d s .
\end{aligned}
$$

Verificamos inicialmente que $F(u)(t, x)$ é limitada em $L^{2}(\Omega)$ para cada $t \in[-T, T]$ e qualquer $u \in X_{T}$. De fato,

$\|F(u)(t, \cdot)\|_{L^{2}(\Omega)}^{2}=\int_{\Omega}\left|e^{-A(x) t} u_{0}(x)+\int_{0}^{t} e^{-A(x)(t-s)} \int_{\Omega} J(x-y) u(s, y) d y d s+\int_{0}^{t} e^{-A(x)(t-s)} f(s, x) d s\right|^{2} d x$.

Usando a desigualdade do Lema 1.2

$$
\begin{aligned}
& \|F(u)(t, \cdot)\|_{L^{2}(\Omega)}^{2} \leq \\
& \int_{\Omega} 4\left(\left|e^{-A(x) t} u_{0}(x)+\int_{0}^{t} e^{-A(x)(t-s)} \int_{\Omega} J(x-y) u(s, y) d y d s\right|^{2}+\left|\int_{0}^{t} e^{-A(x)(t-s)} f(s, x) d s\right|^{2}\right) d x .
\end{aligned}
$$


Novamente, usando o Lema 1.2,

$$
\begin{array}{r}
\|F(u)(t, \cdot)\|_{L^{2}(\Omega)}^{2} \leq \int_{\Omega} 16\left(e^{-2 A(x) t}\left|u_{0}(x)\right|^{2}+\left|\int_{0}^{t} e^{-A(x)(t-s)} \int_{\Omega} J(x-y) u(s, y) d y d s\right|^{2}\right) d x+ \\
+\int_{\Omega} 4\left|\int_{0}^{t} e^{-A(x)(t-s)} f(s, x) d s\right|^{2} d x .
\end{array}
$$

Vamos majorar termo a termo a desigualdade acima. Primeiramente, note que

$$
\max _{(t, x) \in[-T, T] \times \bar{\Omega}} e^{-A(x) t} \leq e^{T \max _{x \in \bar{\Omega}} A(x)} \leq e^{T A_{m}},
$$

onde $A_{m}=\max _{x \in \bar{\Omega}} A(x)$. Dessa maneira, tomando o supremo após usar a Desigualdade de Hölder 1.5 e por (3.15) encontramos

$$
\begin{aligned}
\int_{\Omega} e^{-2 A(x) t}\left|u_{0}(x)\right|^{2} d x & \leq\left\|e^{-2 A(\cdot) t}\right\|_{L^{\infty}(\Omega)}\left\|u_{0}(\cdot)\right\|_{L^{2}(\Omega)}^{2} \\
& \leq e^{2 T A_{m}} \sup _{-T \leq t \leq T}\left\|u_{0}(\cdot)\right\|_{L^{2}(\Omega)}^{2}
\end{aligned}
$$

Seja $K=\int_{\Omega}\left|\int_{0}^{t} e^{-A(x)(t-s)} \int_{\Omega} J(x-y) u(s, y) d y d s\right|^{2} d x$, temos então

$$
K=\left\|\int_{0}^{t} e^{-A(\cdot)(t-s)} \int_{\Omega} J(\cdot-y) u(s, y) d y\right\|_{L^{2}(\Omega)}^{2}
$$

e pela Desigualdade de Hölder 1.5,

$$
K \leq e^{2 T A_{m}}\left\|\int_{0}^{t} 1 \int_{\Omega} J(\cdot-y) u(s, y) d y d s\right\|_{L^{2}(\Omega)}^{2}
$$

Segue que

$$
\begin{aligned}
K & \leq \int_{\Omega} e^{2 T A_{m}}\left[\left(\int_{\Omega} J(x-y)^{2} d y\right)^{\frac{1}{2}}\left(\int_{\Omega}\left(\int_{0}^{t} u(s, y) d s\right)^{2} d y\right)^{\frac{1}{2}}\right]^{2} d x \\
& \leq e^{2 T A_{m}} \int_{\Omega}\|J(x-\cdot)\|_{L^{2}(\Omega)}^{2}\left(\int_{\Omega}|t|\left(\int_{0}^{t} u(s, y)^{2} d s\right) d y\right) d x \\
& \leq e^{2 T A_{m}} T \int_{\Omega}\|J(x-\cdot)\|_{L^{2}(\Omega)}^{2}\left(\int_{0}^{t}\|u(s, \cdot)\|_{L^{2}(\Omega)}^{2} d s\right) d x \\
& \leq e^{2 T A_{m}} T^{2} \sup _{s \in[-T, T]}\|u(s, \cdot)\|_{L^{2}(\Omega)}^{2} \int_{\Omega}\|J(x-\cdot)\|_{L^{2}(\Omega)}^{2} d x \\
& \leq e^{2 T A_{m}} T^{2} \sup _{s \in[-T, T]}\|u(s, \cdot)\|_{L^{2}(\Omega)}^{2}|\Omega|\|J\|_{L^{\infty}(\Omega)}^{2}
\end{aligned}
$$

Agora, seja

$$
R=\int_{\Omega}\left|\int_{0}^{t} e^{-A(\cdot)(t-s)} f(s, \cdot) d s\right|^{2} d x .
$$

Usando a Desigualdade de Hölder 1.5 temos 


$$
\begin{aligned}
R & =\int_{\Omega}\left|\int_{0}^{t} e^{-A(\cdot)(t-s)} f(s, \cdot) d s\right|^{2} d x \\
& \leq \int_{\Omega}\left|e^{2 T A_{m}} \int_{0}^{t} f(s, \cdot) d s\right|^{2} d x \\
& \leq T e^{2 T A_{m}} \sup _{-T \leq s \leq T}\|f(s, \cdot)\|_{L^{2}(\Omega)}^{2} .
\end{aligned}
$$

Juntando todas as informações, temos

$$
\begin{aligned}
\|F(u)(t, \cdot)\|_{L^{2}(\Omega)}^{2} & \leq 16\left(e^{2 T A_{m}} \sup _{-T \leq t \leq T}\left\|u_{0}(\cdot)\right\|_{L^{2}(\Omega)}^{2}+T^{2} e^{2 T A_{m}}\|J\|_{L^{\infty}(\Omega)}^{2}|\Omega| \sup _{-T \leq t \leq T}\|u(s, \cdot)\|_{L^{2}(\Omega)}^{2}\right)+ \\
& +4 T e^{2 T A_{m}} \sup _{-T \leq t \leq T}\|f(s, \cdot)\|_{L^{2}(\Omega)}^{2} .
\end{aligned}
$$

Logo

$$
\sup _{t \in[-T, T]}\|F(u)(s, \cdot)\|_{L^{2}(\Omega)}<\infty .
$$

Agora, a continuidade da $F$ em $t \in[0, T]$ segue diretamente dos Lemas 3.7, 3.8 e do Corolário 3.9. Falta mostrar que $F$ é contração. De fato,

$$
\begin{aligned}
\|F(v)(t, \cdot)-F(w)(t, \cdot)\|_{L^{2}(\Omega)}^{2} & =\left\|e^{-A(\cdot) t} \int_{0}^{t} e^{A(\cdot) s} \int_{\Omega} J(\cdot-y)[v(s, y)-w(s, y)] d y d s\right\|_{L^{2}(\Omega)}^{2} \\
& =\int_{\Omega}\left[e^{-A(x) t} \int_{0}^{t} e^{A(x) s} \int_{\Omega} J(x-y)[v(s, y)-w(s, y)] d y d s\right]^{2} d x
\end{aligned}
$$

Segue analogamente das estimativas para $K$ que

$$
\|F(v)(t, \cdot)-F(w)(t, \cdot)\|_{L^{2}(\Omega)}^{2} \leq T^{2} e^{2 T A_{m}}\|J\|_{L^{\infty}(\Omega)}^{2}|\Omega|^{2}\|v(s, \cdot)-w(s, \cdot)\|_{L^{2}(\Omega)}^{2}, \forall t \in[-T, T]
$$

Assim, podemos escolher $T$ suficientemente pequeno de maneira que $0 \leq T^{2} e^{2 T A_{m}}|\Omega|^{2}\|J\|_{L^{\infty}(\Omega)}^{2}<$ 1, daí segue que $F$ é contração em $X_{T}$. Agora, pelo Teorema 2.3, temos que $F$ possui um único ponto fixo $F(u)(t, x)=u(t, x)$, o que mostra que temos uma única solução do problema em $X_{T}$ para algum $T>0$.

Falta ainda mostrar que $u$ está definida para todo $T>0$. Note que até agora mostramos que $u$ está definida em $[-T, T]$, para $T$ pequeno. Para tanto, considere o mesmo problema, agora com condição inicial $u(T, x)$. Observe que todas as nossas estimativas independem das condições iniciais. Assim conseguimos mostrar existência e unicidade no intervalo $[0,2 T]$. De modo análogo, com condição inicial $u(-T, x)$ obtemos existência e unicidade no intervalo $[-2 T, 0]$. Como $[-2 T, 2 T]=$ $[-2 T, 0] \cup[0,2 T]$, estendemos nossa solução por unicidade para este intervalo. Iterando este processo, temos uma solução definida em $(-\infty,+\infty)=\mathbb{R}$.

Mostremos agora que vale (3.11). Considere

$$
H(t)=\frac{1}{2} \int_{\Omega}\left(u(t, x)-\frac{1}{|\Omega|} \int_{\Omega} u_{0}(x) d s\right)^{2} d x .
$$

Pela Regra da Cadeia temos

$$
H^{\prime}(t)=\int_{\Omega}\left(u(t, x)-\frac{1}{|\Omega|} \int_{\Omega} u_{0}(x) d x\right) u_{t}(t, x) d x .
$$

Mas $u_{t}(t, \cdot)=\int_{\Omega} J(\cdot-y)(u(t, y)-u(t, x) d y+f(t, x)$. Portanto 


$$
H^{\prime}(t)=\int_{\Omega}\left(u(t, x)-\frac{1}{|\Omega|} \int_{\Omega} u_{0}(x) d x\right)\left(\int_{\Omega} J(x-y)(u(t, y)-u(t, x)) d y+f(t, x)\right) d x
$$

Pela Proposição 3.1, usando que $v(x)=u(t, x)-\frac{1}{\Omega} \int_{\Omega} u_{0}(x) d x$, temos

$$
\begin{gathered}
H^{\prime}(t)=-\frac{1}{2} \int_{\Omega} \int_{\Omega} J(x-y)\left(u(t, y)-\frac{1}{|\Omega|} \int_{\Omega} u_{0}(x) d x-\left(u(t, x)-\frac{1}{|\Omega|} \int_{\Omega} u_{0}(x) d x\right)\right) \\
(u(t, y)-u(t, x)) d y d x+\int_{\Omega}\left(u(t, x)-\frac{1}{|\Omega|} \int_{\Omega} u_{0}(x) d x\right) f(t, x) d x
\end{gathered}
$$

Logo

$$
\begin{aligned}
H^{\prime}(t)=-\frac{1}{2} \int_{\Omega} \int_{\Omega} J(x-y)(u(t, y)-u(t, x) & \left.+\frac{1}{|\Omega|} \int_{\Omega} u_{0}(x) d x-\frac{1}{|\Omega|} \int_{\Omega} u_{0}(x) d x\right)^{2} d x+ \\
& +\int_{\Omega}\left(u(t, x)-\frac{1}{|\Omega|} \int_{\Omega} u_{0}(x) d x\right) f(t, x) d x .
\end{aligned}
$$

Seja $W=\left\{u \in L^{2}(\Omega): \int_{\Omega} u(x) d x=0\right\}$, sabemos pela Proposição 3.6 que

$$
\lambda_{1}=\inf _{u \in W} \frac{\frac{1}{2} \int_{\Omega} \int_{\Omega} J(x-y)(u(t, y)-u(t, x))^{2} d y d x}{\int_{\Omega} u^{2}(x) d x}
$$

é positivo. Assim, se $\tilde{u}(t, x)=u(t, x)-\frac{1}{\Omega} \int_{\Omega} u_{0}(x) d x$,

$$
-\lambda_{1} \geq \frac{-\frac{1}{2} \int_{\Omega} \int_{\Omega} J(x-y)(\tilde{u}(t, y)-\tilde{u}(t, x))^{2} d y d x}{\int_{\Omega} \tilde{u}^{2}(x) d x} .
$$

Portanto

$$
\begin{aligned}
-\lambda_{1} \| u(t, x)- & \frac{1}{|\Omega|} \int_{\Omega} u_{0}(x) d x \|_{L^{2}(\Omega)}^{2} \geq \\
& -\frac{1}{2} \int_{\Omega} \int_{\Omega} J(x-y)\left[\left(u(t, y)-\frac{1}{|\Omega|} \int_{\Omega} u_{0}(x) d x\right)-\left(u(t, x)-\frac{1}{|\Omega|} \int_{\Omega} u_{0}(x) d x\right)\right]^{2} d y d x,
\end{aligned}
$$

então

$$
-\lambda_{1} 2 H(t) \geq-\frac{1}{2} \int_{\Omega} \int_{\Omega} J(x-y)(u(t, y)-u(t, x))^{2} d x d y .
$$

Agora, note que no termo $\int_{\Omega}\left(u(t, x)-\frac{1}{|\Omega|} \int_{\Omega} u_{0}(x) d x\right) f(t, x) d x$ de $H^{\prime}(t)$, podemos usar a Desigualdade de Young (1.3),

$$
\frac{a}{\sqrt{2} \delta} b \sqrt{2} \delta \leq \frac{a^{2}}{4 \delta^{2}}+b^{2} \delta^{2}
$$

onde $a=f(t, x)$ e $b=u(t, x)-\frac{1}{\Omega} \int_{\Omega} u_{0}(x) d x$, temos 


$$
\int_{\Omega}\left(u(t, x)-\frac{1}{|\Omega|} \int_{\Omega} u_{0}(x) d x\right) f(t, x) d x \leq \int_{\Omega} \frac{f^{2}(t, x)}{4} d x \delta^{-2}+\int_{\Omega}\left(u(t, x)-\frac{1}{\Omega} \int_{\Omega} u_{0}(x) d x\right)^{2} \delta^{2} .
$$

Destas informações, obtemos

$$
H^{\prime}(t) \leq-\lambda_{1} 2 H(t)+\frac{\|f(t, .)\|_{L^{2}(\Omega)}^{2}}{4} \delta^{-2}+\delta^{2} 2 H(t) .
$$

Portanto

$$
\begin{aligned}
H^{\prime}(t) & \leq 2\left(\delta^{2}-\lambda_{1}\right) H(t)+\frac{\delta^{-2}}{4}\|f(t, \cdot)\|_{L^{2}(\Omega)}^{2} \\
& \leq 2\left(\delta^{2}-\lambda_{1}\right) H(t)+\delta^{-2}\|f(t, \cdot)\|_{L^{2}(\Omega)}^{2}
\end{aligned}
$$

Integrando $H(t)$, obtemos

$$
H(t) \leq e^{2\left(\delta^{2}-\lambda_{1}\right)}\left[H(0)+\delta^{-2} \int_{0}^{t}\|f(s, \cdot)\|_{L^{2}(\Omega)}^{2} d s\right] .
$$

Agora, tomando $\delta$ pequeno o suficiente para que $\delta^{2}-\lambda_{1}<0$, concluímos que

$$
\left\|u(t, \cdot)-\frac{1}{\Omega} \int_{\Omega} u_{0}(x) d x\right\|_{L^{2}(\Omega)}^{2} \leq e^{-\alpha t}\left[\left\|u_{0}(x)-\frac{1}{\Omega} \int_{\Omega} u_{0}(x) d x\right\|_{L^{2}(\Omega)}^{2}+\delta^{-1} \int_{0}^{t}\|f(s, \cdot)\|_{L^{2}(\Omega)}^{2} d s\right],
$$

onde $2\left(\delta^{2}-\lambda_{1}\right)=-\alpha$ e $\delta^{-2}=C$.

Mostremos agora (3.14).

Chamando

$$
w(t, x)=u(t, x)-\frac{1}{\Omega} \int_{\Omega} u_{0}(x) d x,
$$

$w$ satisfaz (3.1), com condição inicial $w_{0}(x)=u_{0}(x)-\frac{1}{\Omega} \int_{\Omega} u_{0}(x) d x$, e então a solução fica

$$
w(t, x)=e^{-A(x) t}\left[w_{0}+\int_{0}^{t} e^{A(x) s} f(s, x) d s+\int_{0}^{t} e^{A(x) s} \int_{\Omega} J(x-y) w(s, y) d y d s\right] .
$$

Segue, pela Desigualdade de Hölder 1.5

$$
|w(t, x)| \leq e^{-A(x) t}\left[\left|w_{0}\right|+\int_{0}^{t} e^{A(x) t}|f(s, x)| d s+\int_{0}^{t}\|J(x-\cdot)\|_{L^{2}(\Omega)}\|w(s, \cdot)\|_{L^{2}(\Omega)} d s\right] .
$$

Como $J$ é contínua, $\mathrm{J}(0)>0$ e $\Omega$ é um domínio limitado, existe $m>0$ constante tal que

$$
0<m \leq A(x)=\int_{\Omega} J(x-y) d y \leq\|J\|_{\infty}|\Omega|=\gamma
$$

pois a extensão de $A(x)$ até $\bar{\Omega}$ é contínua, logo $A(x)$ admite mínimo.

Como $\|J(x-\cdot)\|_{L^{2}(\Omega)} \leq\left|\left\|\left.J(x-\cdot)\right|^{2}\right\|_{L^{\infty}(\Omega)}\right.$, segue que, por (3.11), (3.12), (3.13) e pela Desigualdade de Hölder 1.5 


$$
\begin{aligned}
& |w(t, x)| \leq e^{-A(x) t}\left|w_{0}\right|+e^{-A(x) t} \int_{0}^{t} e^{A(x) s}|f(s, x)| d s+ \\
& +e^{-A(x) t} \int_{0}^{t} e^{A(x) s}\|J(x-\cdot)\|_{L^{2}(\Omega)} e^{-\alpha s}\left(\left\|w_{0}\right\|_{L^{2}(\Omega)}+e^{-A(x) t} C \int_{0}^{t}\|f(\tau, \cdot)\|_{L^{2}(\Omega)}^{2} d \tau\right) d s \\
& |w(t, x)| \leq e^{-A(x) t}\left|w_{0}\right|+e^{-A(x) t} \int_{0}^{\infty} e^{\gamma s}|f(s, x)| d s+ \\
& +e^{-A(x) t}\|J\|_{L^{\infty}(\Omega)}|\Omega|^{\frac{1}{2}} \int_{0}^{t} e^{(A(x)-\alpha) s}\left(\left\|w_{0}\right\|_{L^{2}(\Omega)}+C \int_{0}^{\infty}\|f(\tau, \cdot)\|_{L^{2}(\Omega)}^{2} d \tau\right) d s \\
& |w(t, x)| \leq e^{-A(x) t}\left|w_{0}\right|+e^{-A(x) t} \int_{0}^{\infty} e^{\gamma s}|f(s, x)| d s+ \\
& +\|J\|_{L^{\infty}(\Omega)}|\Omega|^{\frac{1}{2}} e^{-A(x) t}\left(\frac{e^{(A(x)-\alpha) t}-1}{A(x)-\alpha}\right)\left(\left\|w_{0}\right\|_{L^{2}(\Omega)}+C \int_{0}^{\infty}\|f(\tau, \cdot)\|_{L^{2}(\Omega)}^{2} d \tau\right) .
\end{aligned}
$$

Assim, podemos tomar $\alpha>0$ pequeno o suficiente em (3.11) tal que, para algum $m_{1}>0$,

$$
0<m_{1} \leq A(x)-\alpha \leq \gamma .
$$

Então,

$$
\begin{aligned}
|w(t, x)| \leq & e^{-m t}\left|w_{0}(x)\right|+e^{-m t} \int_{0}^{\infty} e^{\gamma s}|f(s, x)| d s+ \\
& +e^{-\alpha t}\left(\frac{\|J\|_{L^{\infty}(\Omega)}|\Omega|^{\frac{1}{2}}}{m_{1}}\right)\left(\left\|w_{0}\right\|_{L^{2}(\Omega)}+C \int_{0}^{\infty}\|f(\tau, \cdot)\|_{L^{2}(\Omega)}^{2} d \tau\right) .
\end{aligned}
$$

Temos então

$$
\begin{aligned}
|w(t, x)| & \leq e^{-m t} c_{1}+e^{-\alpha t} c_{2} \\
& \leq e^{-\alpha t}\left(\frac{e^{-m t c_{1}}}{e^{-\alpha t}}+c_{2}\right) \\
& \leq e^{-\alpha t} e^{t(\alpha-m)} c_{1}+c_{2} \\
& \leq e^{-\alpha t} c_{3} .
\end{aligned}
$$

Tomando o supremo em $x$

$$
\|w(t, \cdot)\|_{L^{\infty}(\Omega)} \leq e^{-\alpha t} c_{3} .
$$

O que nos dá o que queríamos.

\subsection{Uma Equação Semi-Linear}

Vamos considerar a partir de agora, o seguinte problema não local semilinear

$$
\left\{\begin{array}{l}
u_{t}(t, x)=\int_{\Omega} J(x-y)(u(t, y)-u(t, x)) d y+f(u(t, x)), \\
u(0, x)=u_{0}(x),
\end{array}\right.
$$

com $x \in \Omega, t>0, J$ satisfazendo $(\mathbf{H})$ e $f: \mathbb{R} \longrightarrow \mathbb{R}$ globalmente Lipschitz.

Teorema 3.11 Se $u_{0} \in L^{2}(\Omega)$, o problema (3.16) possui uma única solução global

$$
u: \mathbb{R} \times \Omega \longrightarrow \mathbb{R}
$$


tal que $u \in C^{1}\left([a, b], L^{2}(\Omega)\right)$, com

$$
u(t, x)=e^{-A(x) t} u_{0}+\int_{0}^{t} e^{-A(x)(t-s)} \int_{\Omega} J(x-y) u(s, y) d y d s+\int_{0}^{t} e^{-A(x)(t-s)} f(u(s, x)) d s,
$$

para $(t, x) \in \mathbb{R} \times \Omega$, onde $A(x) \in L^{\infty}(\Omega)$ é a função positiva, já definida anteriormente,

$$
A(x)=\int_{\Omega} J(x-y) d y, x \in \Omega .
$$

Demonstração: Observe primeiramente que a equivalência de (3.17) e do problema (3.16) é análoga ao que foi feito no Teorema 3.10. Trabalharemos com $[a, b]=[-T, T]$.

Consideremos $T>0$ fixo no espaço de Banach

$$
X_{T}=\left\{u \in C\left([-T, T], L^{2}(\Omega)\right): \sup _{-T \leq t \leq T}\|u(t, \cdot)\|_{L^{2}(\Omega)}<\infty\right\} .
$$

A solução do problema (3.16) será dada, como no Teorema 3.10, como um ponto fixo da aplicação

$$
F: X_{T} \longrightarrow X_{T}
$$

definida por

$$
\begin{aligned}
F(u)(t, x) & =e^{-A(x) t} u_{0}+\int_{0}^{t} e^{-A(x)(t-s)} \int_{\Omega} J(x-y) u(s, y) d y d s+ \\
& +\int_{0}^{t} e^{-A(x)(t-s)} f(u(s, x)) d s .
\end{aligned}
$$

Mostremos que $F$ é limitada em $X_{T}$. De fato,

$\|F(u)(t, \cdot)\|_{L^{2}(\Omega)}^{2}=\int_{\Omega}\left|e^{-A(x) t} u_{0}+\int_{0}^{t} e^{-A(x)(t-s)} \int_{\Omega} J(x-y) u(s, y) d y d s+\int_{0}^{t} e^{-A(x)(t-s)} f(u(s, x)) d s\right|^{2} d x$

Pelo Lema 1.2

$$
\begin{aligned}
\|F(u)(t, \cdot)\|_{L^{2}(\Omega)}^{2} & \leq \int_{\Omega} 4\left|e^{-A(x) t} u_{0}+\int_{0}^{t} e^{-A(x)(t-s)} \int_{\Omega} J(x-y) u(s, y) d y d s\right|^{2} d x+ \\
& +\int_{\Omega} 4\left|\int_{0}^{t} e^{-A(x)(t-s)} f(u(s, x)) d s\right|^{2} d x .
\end{aligned}
$$

Usando o Lema 1.2 novamente,

$$
\begin{aligned}
\|F(u)(t, \cdot)\|_{L^{2}(\Omega)}^{2} & \leq \int_{\Omega} 16\left[\left|e^{-A(x) t} u_{0}\right|^{2}+\left|\int_{0}^{t} e^{-A(x)(t-s)} \int_{\Omega} J(x-y) u(s, y) d y d s\right|^{2}\right] d x+ \\
& +4 \int_{\Omega}\left|\int_{0}^{t} e^{-A(x)(t-s)} f(u(s, x))\right|^{2} d x .
\end{aligned}
$$

Sabemos que

$$
\int_{\Omega}\left|e^{-A(x) t} u_{0}\right|^{2} d x \leq e^{2 T A_{m}} \sup _{-T \leq t \leq T}\left\|u_{0}(\cdot)\right\|_{L^{2}(\Omega)}^{2}
$$

e

$$
K=\left|\int_{0}^{t} e^{-A(x)(t-s)} \int_{\Omega} J(x-y) u(s, y) d y d s\right|^{2} \leq T^{2} e^{2 T A_{m}}\|J\|_{L^{\infty}(\Omega)}^{2}|\Omega|^{2} \sup _{0 \leq s \leq T}\|u(s, \cdot)\|_{L^{2}(\Omega)}^{2} .
$$


Falta majorarmos o termo

$$
4 \int_{\Omega}\left|\int_{0}^{t} e^{-A(x)(t-s)} f(u(s, x)) d s\right|^{2} d x .
$$

Mas, sabemos que, utilizando a Desigualdade de Hölder 1.5 e (3.15)

$$
4 \int_{\Omega} \mid \int_{0}^{t} e^{-A(x)(t-s)} f\left(\left.u(s, x) d s\right|^{2} d x \leq 4 T^{2} e^{2 T A_{m}} \sup _{-T \leq s \leq T}\|f(u(s, \cdot))\|_{L^{2}(\Omega)}^{2} .\right.
$$

A continuidade de $F$ em $t \in[-T, T]$ segue de modo análogo ao que foi feito no Teorema 3.10. Assim, como $f$ é globalmente Lipschitz, concluímos de tais desigualdades que $F$ está bem definida, i.e, $F(u) \in X_{T}$ sempre que $u \in X_{T}$.

Agora falta mostrarmos que $F$ é contração, de fato

$$
\begin{aligned}
\|F(v)(t, \cdot)-F(w)(t, \cdot)\|_{L^{2}(\Omega)}^{2}= & \| e^{-A(\cdot)} u_{0}+\int_{0}^{t} e^{-A(\cdot)(t-s)} J(\cdot-y) v(t, y) d y d s+ \\
& +\int_{0}^{t} e^{-A(\cdot)(t-s)} f(v(s, \cdot)) d s- \\
& -e^{-A(\cdot)} u_{0}-\int_{0}^{t} e^{-A(\cdot)(t-s)} J(\cdot-y) w(t, y) d y d s- \\
& -\int_{0}^{t} e^{-A(\cdot)(t-s)} f(w(s, \cdot)) d s \|_{L^{2}(\Omega)}^{2} \\
\|F(v)(t, \cdot)-F(w)(t, \cdot)\|_{L^{2}(\Omega)}^{2}=\| e^{-A(\cdot)} u_{0}+\int_{0}^{t} e^{-A(\cdot)(t-s)} J(\cdot-y)(v(t, y)-w(s, y) d y d s+ & +\int_{0}^{t} e^{-A(\cdot)(t-s)}(f(v(s, \cdot))-f(w(s, \cdot))) d s \|_{L^{2}(\Omega)}^{2} \cdot
\end{aligned}
$$

Aplicando o Lema 1.2

$$
\begin{aligned}
\|F(v)(t, \cdot)-F(w)(t, \cdot)\|_{L^{2}(\Omega)}^{2} \leq & 4\left\|e^{-A(\cdot) t} \int_{0}^{t} e^{A(\cdot) s} \int_{\Omega} J(\cdot-y)(v(s, y)-w(s, y)) d y d s\right\|^{2}+ \\
& +4 \| \int_{0}^{t} e^{A(\cdot)(t-s)}\left(f(v(s, \cdot)-w(s, \cdot)) d s \|^{2} \cdot\right.
\end{aligned}
$$

De modo análogo ao que foi feito no Teorema 3.10, concluímos que

$$
\begin{aligned}
\|F(v)(t, \cdot)-F(w)(t, \cdot)\|_{L^{2}(\Omega)}^{2} & \leq 4 T^{2} e^{2 T A_{m}}\|J\|_{L^{\infty}(\Omega)}^{2}|\Omega|^{2}\|v(s, \cdot)-w(s, \cdot)\|_{L^{2}(\Omega)}^{2}+ \\
& +\| \int e^{-A(\cdot)(t-s)}\left(f(v(s, \cdot)-f(w(s, \cdot))) d s \|_{L^{2}(\Omega)}^{2} .\right.
\end{aligned}
$$

Mas, como $f$ é globalmente Lipschitz, sabemos que

$$
\begin{aligned}
\| \int e^{-A(\cdot)(t-s)}\left(f(v(s, \cdot)-f(w(s, \cdot))) d s \|_{L^{2}(\Omega)}^{2}\right. & \leq T^{2} e^{2 T A_{m}} \| f\left(v(s, \cdot)-f(w(s, \cdot)) \|_{L^{2}(\Omega)}^{2}\right. \\
& \leq T^{2} k^{2}\|v(s, \cdot)-w(s \cdot)\|_{L^{2}(\Omega)}^{2}
\end{aligned}
$$

onde $k$ é a constante de Lipschitz.

Portanto, temos 


$$
\begin{aligned}
\|F(v)(t, \cdot)-F(w)(t, \cdot)\|_{L^{2}(\Omega)}^{2} & \leq\left(4|\Omega|^{2} T^{2} e^{2 T A_{m}}+T^{2} e^{2 T A_{m}} k^{2}\right)\|v(t, \cdot)-w(t, \cdot)\|_{L^{2}(\Omega)}^{2} \\
& \leq\left(4|\Omega|^{2}+k^{2}\right) T^{2} e^{2 T A_{m}}\|v(t, \cdot)-w(t, \cdot)\|_{L^{2}(\Omega)}^{2} .
\end{aligned}
$$

Se tomarmos

$$
T^{2}<\frac{1}{\left(4|\Omega|^{2}+k^{2}\right) e^{2 T A_{m}}},
$$

temos uma contração, portanto segue pelo Teorema 2.1 que existe um único ponto fixo para $F(u)(t, x)=u(t, x)$. Mais uma vez, de modo análogo ao que foi feito no Teorema 3.10, conseguimos estender $u$ para toda a reta $\mathbb{R}$ obtendo uma única solução $u \in C\left((-\infty,+\infty), L^{2}(\Omega)\right)$

\subsection{Regularidade com Relação aos Parâmetros}

Nesta seção, consideremos uma bola $B_{r}(0)$, com raio $r$ suficientemente grande centrada na origem de tal maneira que $x-y \in B_{r}(0)$ para todo $x, y \in \Omega$. Além disso consideramos o conjunto

$$
Z=\left\{J \in C\left(\overline{B_{r}(0)}, \mathbb{R}\right): J \geq 0, J(0) \geq c>0, J(-x)=J(x), \text { e } J(x)=0 \text { em } \mathbb{R}^{n} \backslash B_{r}(0)\right\} .
$$

Como na seção anterior, assumimos $f: \mathbb{R} \longrightarrow \mathbb{R}$ globalmente Lipschitz. A partir disso, consideremos a aplicação $F$ da seguinte maneira

$$
F: X_{T} \times L^{2}(\Omega) \times Z \longrightarrow X_{T}
$$

onde $X_{T}=\left\{u \in C\left([-T, T], L^{2}(\Omega)\right): \sup _{-T \leq t \leq T}\|u(t, \cdot)\|_{L^{2}(\Omega)}<\infty\right\}$ e

$$
F\left(u, u_{0}, J\right)=e^{-A(x) t} u_{0}(x)+\int_{0}^{t} e^{-A(x)(t-s)} \int_{\Omega} J(x-y) u(s, y) d y d s+\int_{0}^{t} e^{-A(x)(t-s)} f(u(s, x)) d s .
$$

Proposição 3.12 O espaço $Z$ munido da norma

$$
\|J\|=\sup _{x \in B_{r}(0)}|J(x)|
$$

é um espaço de Banach.

Demonstração: Seja $J_{n}$ uma sequência de Cauchy em $Z$. Então, dado $\varepsilon>0$, existe $n_{0}>0$ tal que, se $m, n>n_{0}$, então

$$
\left\|J_{n}-J_{m}\right\|<\varepsilon
$$

Logo, para todo $x \in B_{r}(0)$ temos

$$
\left|J_{n}(x)-J_{m}(x)\right| \leq \sup _{x \in B_{r}(0)}\left|J_{n}(x)-J_{m}(x)\right|=\left\|J_{n}-J_{m}\right\|<\varepsilon .
$$

Então para todo $x$, temos que $J_{n}(x)$ é de Cauchy em $\mathbb{R}$. Assim podemos concluir que $J_{n}$ é pontualmente convergente.

Seja $J: B_{r}(0) \longrightarrow \mathbb{R}$ definida por

$$
J(x)=\lim _{n \rightarrow \infty} J_{n}(x) .
$$

Afirmamos que $J \in Z$. De fato, como $J_{n}$ é par, temos que $J$ é par, e mais, $J(0) \geq c$ e $J \geq 0$, pois $J_{n}$ também é.

Mostremos agora que $J$ é contínua. Note que $J_{n}$ é contínua definida no compacto $\overline{B_{r}(0)}$, logo é uniformemente contínua. Assim, pelo Teorema de Dini (consultar obra [7], página 211), que afirma que o limite de funções uniformemente contínuas é contínua, obtemos que $J$ é contínua. 
Falta-nos mostrar que $J_{n} \rightarrow J$ uniformemente. Dado $\varepsilon>0$, existe $n_{1}>0$ tal que se $n>n_{1}$ então $\left\|J_{n}-J\right\|<\varepsilon$. De fato

$$
\begin{aligned}
\left|J_{n}(x)-J_{n}(y)\right| & =\left|J_{n}(x)-J_{m}(x)+J_{m}(x)-J_{m}(y)+J_{m}(y)-J_{n}(y)\right| \\
& <\varepsilon+\left|J_{m}(x)-J_{m}(y)\right|+\varepsilon,
\end{aligned}
$$

Mas como $J_{m}$ é uniformemente contínua, temos que $\left|J_{m}(x)-J_{m}(y)\right|<\varepsilon$ para todo $x, y$ com $|x-y|<\delta$, para algum $\delta>0$.

Assim, como $n$ é arbitrário, segue que $\left\{J_{n}\right\}$ é equicontínua. Como também sabemos que $J_{n} \rightarrow J$ ponto a ponto, temos também que é pontualmente limitada.

Segue, pelo Teorema de Arzelá-Ascoli 1.4, que $J_{n} \rightarrow J$ uniformemente.

Temos então que $J_{n} \rightarrow J$ uniformemente, e segue então que $Z$ é espaço de Banach.

Proposição 3.13 A aplicação $F$ definida em (3.18) é da forma

$$
F\left(u, u_{0}, J\right)=L\left(u, u_{0}, J\right)+B(u),
$$

onde $L: X_{T} \times L^{2}(\Omega) \times Z \longrightarrow X_{T}$ é uma aplicação afim dada por

$$
L\left(u, u_{0}, J\right)=e^{-A(x) t} u_{0}(x)+\int_{0}^{t} e^{-A(x)(t-s)} \int_{\Omega} J(x-y) u(s, y) d y d s
$$

e $B: X_{T} \longrightarrow X_{T}$ é o funcional definido por

$$
B u=\int_{0}^{t} e^{-A(x)(t-s)} f(u(s, x)) d s .
$$

Além disso, temos os seguintes casos:

- Se $B=0$, a solução u do problema linear

$$
\left\{\begin{array}{l}
u_{t}(t, x)=\int_{\Omega} J(x-y)(u(t, y)-u(t, x)) d y \\
u(0, x)=u_{0}(x)
\end{array}\right.
$$

é analítica como uma função de $L^{2}(\Omega) \times Z \longrightarrow X_{T}:\left(u_{0}, J\right) \longmapsto u\left(u_{0}, J\right)$.

- Se $B \neq 0$, a solução u de (3.16) é contínua nos mesmos espaços.

Demonstração: Queremos mostrar que $F\left(u, u_{0}, J\right)$ satisfaz as hipóteses do Teorema 2.3. Sabemos dos Lemas 3.7, 3.8 e Corolário 3.9 que os termos

$$
e^{-A(x) t} u_{0}(x), \int_{0}^{t} e^{-A(x)(t-s)} \int_{\Omega} J(x-y) u(s, y) d y d s \text { e } \int_{0}^{t} e^{-A(x)(t-s)} f(u(s, x)) d s
$$

são uniformemente contínuos e portanto, contínuos. Daí concluímos que $F\left(u, u_{0}, J\right)$ está bem definida.

De modo análogo ao que foi feito no Teorema 3.10, também obtemos que $F\left(u, u_{0}, J\right)$ é contração uniforme com:

$$
\left\|F\left(v, u_{0}, J\right)(t, \cdot)-F\left(w, u_{0}, J\right)(t, \cdot)\right\|_{L^{2}(\Omega)}^{2} \leq\left(4|\Omega|^{2}+k^{2}\right) T^{2} e^{2 T A_{m}}\|J\|_{L^{\infty}(\Omega)}^{2}\|v(t, \cdot)-w(t, \cdot)\|_{L^{2}(\Omega)}^{2}
$$

assumindo $T^{2}<\frac{1}{\left(4|\Omega|^{2}+k^{2}\right) e^{2 T A_{m}}}$.

Com tais condições satisfeitas temos então que $F\left(u, u_{0}, J\right)$ possui um único ponto fixo, $u=$ $u\left(u_{0}, J\right)$, dependendo da condição inicial $u_{0}$ e o kernel do operador integral $J$. 
Agora, se $B \equiv 0$, temos que $F$ é da forma

$$
F\left(u, u_{0}, J\right)=L\left(u, u_{0}, J\right),
$$

donde segue que $F$ é analítica de $X_{T} \times L^{2}(\Omega) \times Z$ em $X_{T}$, pois sabemos que toda função afim é analítica. Pelo Teorema 2.3, concluímos que $u$ é analítica em seu domínio.

Se $B \neq 0$, como $f$ é globalmente Lipschitz, obtemos que $B$ está bem definida e também é globalmente Lipschitz. Temos então que $B$ é contínua e, pelo Teorema 2.3, obtemos que $u$ é contínua. 
PROBLEMA DE NEUMANN NÃO LOCAL 


\section{Referências Bibliográficas}

[1] Andreu-Vaillo, F.; Mazón, J. M.; Rossi, J. D.; Toledo, J. J.; Evolution Problems with Nonlocal Diffusion, Mathematics Subject Classification, Argentina, 1991. 1, 4, 29

[2] Brezis, H.; Funcional Analysis, Sobolev Spaces and Partial Differential Equations, Springer, New York, 2010. 12, 13

[3] Carvalho, A. N., Análise Funcional II, Notas de Aula, 2016, disponível em http://conteudo.icmc.usp.br/pessoas/andcarva/AnaliseFuncional-II/AnaliseFuncional-II.pdf, acessado em Agosto de 2017.

[4] Fife, P. Some nonclassical trends in parabolic and parabolic-like evolutions, Trends in non-linear analysis, 153;191, Springer, Berlin, 2003. iii, v, 1, 4

[5] Henry, D. B.; Geometric Theory of Semilinear Parabolic Equations, Springer, New York, 1981. 13

[6] Kreyzig, E. Introdutory Functional Analysis with Aplications, John Wiley and Sons, 1979.

[7] Lima, E. L.; Espaços Métricos, Terceira Edição, Projeto Euclides, IMPA, Rio de Janeiro, 1993. 47

[8] Murray, J. D.; Mathematical Biology I: An introduction, 3rd edition, Springer, New York, 2002. 3

[9] Oliveira, O. R. B.; EDP's Elípticas, Notas de aula, 2017, disponível em https://www.ime.usp.br/ oliveira/Disciplinas.html, acessado em Novembro de 2017. 32

[10] Pereira, M. C., Rossi, J. D.; Nonlocal Evolution Problems in Thin Domains, 1, 29

[11] Rudin, W.; Functional Analysis, Segunda Edição, 1976, McGrall-Hill, 1991. 5

[12] Sastre, S.; Nonlocal Diffusion Problems, Tese de Doutorado - Matemática Aplicada, Universidad Complutense de Madrid, Madrid, 2014. 